OPEN ACCESS

Edited by:

Wei Yang,

State College of Florida,

Manatee-Sarasota, USA

Reviewed by:

Vladimir N. Uversky,

University of South Florida, USA

Ray Luo,

University of California, Irvine, USA Mikolai Fajer,

UT-Battelle, USA

*Correspondence:

Robert Schneider

robert.schneider@univ-lille1.fr

Specialty section:

This article was submitted to

Molecular Recognition,

a section of the journal

Frontiers in Molecular Biosciences

Received: 18 July 2016 Accepted: 24 August 2016 Published: 09 September 2016

Citation:

Mollica L, Bessa LM, Hanoulle X

Jensen MR, Blackledge $M$ and

Schneider R (2016) Binding

Mechanisms of Intrinsically Disordered

Proteins: Theory, Simulation, and

Experiment. Front. Mol. Biosci. 3:52.

doi: $10.3389 /$ fmolb.2016.00052

\section{Binding Mechanisms of Intrinsically Disordered Proteins: Theory, Simulation, and Experiment}

\author{
Luca Mollica ${ }^{1}$, Luiza M. Bessa ${ }^{2}$, Xavier Hanoulle², Malene Ringkjøbing Jensen ${ }^{3}$, \\ Martin Blackledge ${ }^{3}$ and Robert Schneider ${ }^{2 *}$
}

${ }^{1}$ CompuNet, Drug Discovery and Development, Istituto Italiano di Tecnologia, Genova, Italy, ${ }^{2}$ NMR \& Molecular Interactions, Université de Lille, CNRS, UMR 8576 - UGSF - Unité de Glycobiologie Structurale et Fonctionnelle, Lille, France, ${ }^{3}$ Institut de Biologie Structurale, CEA, CNRS, Université Grenoble Alpes, Grenoble, France

In recent years, protein science has been revolutionized by the discovery of intrinsically disordered proteins (IDPs). In contrast to the classical paradigm that a given protein sequence corresponds to a defined structure and an associated function, we now know that proteins can be functional in the absence of a stable three-dimensional structure. In many cases, disordered proteins or protein regions become structured, at least locally, upon interacting with their physiological partners. Many, sometimes conflicting, hypotheses have been put forward regarding the interaction mechanisms of IDPs and the potential advantages of disorder for protein-protein interactions. Whether disorder may increase, as proposed, e.g., in the "fly-casting" hypothesis, or decrease binding rates, increase or decrease binding specificity, or what role pre-formed structure might play in interactions involving IDPs (conformational selection vs. induced fit), are subjects of intense debate. Experimentally, these questions remain difficult to address. Here, we review experimental studies of binding mechanisms of IDPs using NMR spectroscopy and transient kinetic techniques, as well as the underlying theoretical concepts and numerical methods that can be applied to describe these interactions at the atomic level. The available literature suggests that the kinetic and thermodynamic parameters characterizing interactions involving IDPs can vary widely and that there may be no single common mechanism that can explain the different binding modes observed experimentally. Rather, disordered proteins appear to make combined use of features such as pre-formed structure and flexibility, depending on the individual system and the functional context.

Keywords: intrinsically disordered proteins, protein-protein interactions, nuclear magnetic resonance, kinetics, molecular dynamics simulations

\section{INTRODUCTION}

The discovery of intrinsically disordered proteins (IDPs) has considerably enhanced our view of protein structure and function. Over the last two decades, it has become accepted that proteins can be functional in the absence of a stable three-dimensional structure (Wright and Dyson, 1999; Dunker et al., 2001, 2002; Tompa, 2002; Dyson and Wright, 2005), and more recently, it has been shown that intrinsic disorder is compatible with the environment inside the cell 
(Bodart et al., 2008; Theillet et al., 2016). Bioinformatic predictions estimate that on the order of $30 \%$ of eukaryotic proteins contain disordered regions of sizable length $(>=50$ residues; Dunker et al., 2000), and the DisProt database of protein disorder (http://www.disprot.org/) now contains entries for 1539 disordered protein regions and 694 entirely disordered proteins (Sickmeier et al., 2007). Their abundance, as well as their implication in disease (Uversky et al., 2008), has sparked considerable interest in IDPs, and a large number of studies have been devoted to the development of experimental and computational approaches in order to understand their conformational behavior and molecular function (Jensen et al., 2014).

IDPs are implicated in a wide range of biological functions, among them notably signal transduction, scaffolding, transcription, cell cycle regulation, or chaperoning (Dunker et al., 2002; Dyson and Wright, 2005). A common theme to these functions are interactions with other proteins or alternatively DNA or small molecules. Given the apparent frequency with which disorder occurs in the aforementioned functional contexts, questions arise as to how IDPs interact with their partners in the absence of well-structured binding sites, which mechanisms they employ to assure specific binding, and in general the advantages intrinsic disorder may have for protein-protein interactions.

In many cases, IDPs do not exhibit fully random statistical coil behavior, but can adopt transiently populated secondary structures and long-range tertiary interactions (Fuxreiter et al., 2004; Salmon et al., 2010). Such structural preorganization has often been found relevant in intermolecular interactions undergone by IDPs. While so-called "fuzzy complexes" of IDPs have been described that retain a high degree of disorder even in the bound state (Tompa and Fuxreiter, 2008), binding of IDPs to physiological partners is often accompanied by a gain in structuration of the binding region, a phenomenon known as "folding upon binding" or "coupled folding and binding" (Dyson and Wright, 2002). How exactly this is accomplished mechanistically has been the subject of intense debate. The discussion has mostly focused on two mechanisms that can be considered as limiting cases, conformational selection (i.e., folding before binding; Karush, 1950; Ma et al., 1999) and induced fit (i.e., folding after binding; Koshland, 1958; Figure 1), while more complex mechanisms such as different combinations of these two have also been envisioned (Csermely et al., 2010). In parallel, other, partially related questions have been discussed, such as whether increasing disorder speeds up binding, as proposed in the often-cited "fly-casting" hypothesis (Shoemaker et al., 2000), or conversely, whether increased pre-structuration of IDP binding sites allows for faster binding (Iešmantavičius et al., 2014). Another matter of debate concerns binding affinities and binding specificity achievable in the context of intrinsic disorder, with IDPs often said to accomplish highly specific binding without concomitant high affinity, which may be of advantage in the context of signaling where a once-formed complex needs to dissociate again to switch off the corresponding signal (Tompa, 2002; Zhou, 2012).

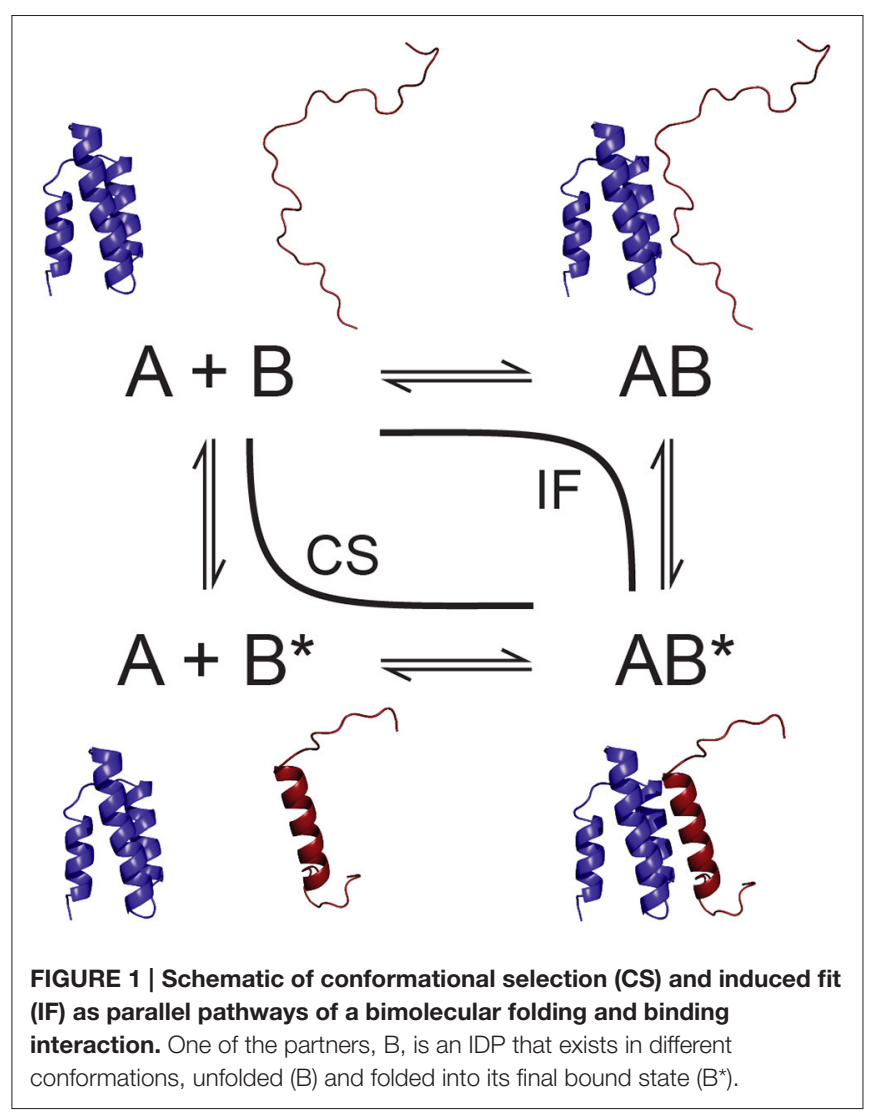

Answering these questions is not a trivial task, and especially obtaining detailed mechanistic information on binding interactions, which usually requires the extraction of the rate constants involved, is notoriously difficult. Correspondingly, the number of experimental studies explicitly addressing IDP binding mechanisms is still relatively limited. Nevertheless, recent years have seen a steady increase of research into the details of IDP binding interactions. Two experimental techniques have in particular been used, namely nuclear magnetic resonance (NMR) spectroscopy and non-equilibrium transient kinetic techniques, such as stopped flow or temperature jump experiments (Gianni et al., 2016). Surface plasmon resonance (SPR) measurements also allow for detailed insight into binding kinetics at high temporal resolution, however at the price of immobilization of one of the binding partners, which may affect the interaction in the case of a highly flexible IDP (Schuck and Zhao, 2010). In addition, single-molecule experiments such as fluorescence resonance energy transfer (Gambin et al., 2011) or nanopore translocation (Japrung et al., 2013) have recently gained importance in studying binding reactions. In parallel to these experimental techniques, modeling and computer simulations have increasingly contributed to our understanding of protein interactions at the atomic level, notably also due to the development of various enhanced sampling techniques (Zhou and Bates, 2013; De Vivo et al., 2016). Here, we have chosen to review recent progress in the field based on NMR spectroscopy and transient kinetics experiments, as well as molecular simulations. 


\section{METHODOLOGY}

\section{Transient Kinetics}

Kinetic information on a binding interaction can be obtained from non-equilibrium techniques that either rapidly mix the reactants (e.g., stopped or continuous flow) or perturb a preexisting equilibrium between them, for example by application of a rapid temperature or pressure jump, and then follow the (re-)establishment of equilibrium via a signal whose variation is related to the binding reaction. Usually, optical signals are employed, such as circular dichroism (CD), absorbance, or the fluorescence of a native or introduced aromatic residue, which vary in the course of a folding and/or binding reaction (Bernasconi, 1976; Figure 2A). These techniques, originally developed in the context of the study of enzymatic reactions (Eigen and Hammes, 1963), are sensitive to processes occurring on the timescales of microseconds (temperature or pressure jump, continuous flow) to milliseconds and longer (stopped flow) (Gianni et al., 2016). Depending on the relaxation time course of the signal monitored, one or more kinetic time constants ( $\lambda$ or $k_{\text {obs }}$ ) are obtained from fitting the signal decay. For a simple two-state bimolecular binding reaction with 1:1 stoichiometry

$$
\mathrm{A}+\mathrm{B} \underset{k_{\mathrm{off}}}{\stackrel{k_{\mathrm{on}}}{\rightleftharpoons}} \mathrm{AB}
$$

assuming pseudo-first-order conditions for A (i.e., B is present in excess), $k_{\text {obs }}$ is given by the sum of the forward and reverse reaction rate constants, $k_{\mathrm{on}}[\mathrm{B}]+k_{\text {off. }}$. A multiexponential decay, on the other hand, is direct evidence for a more complex binding mechanism (Kiefhaber et al., 2012; Vogt and Di Cera, 2012). The reaction is then followed over a range of concentrations of the binding partner in excess. Fitting appropriate models to the variation of the observed relaxation rate constant(s) with binding partner concentration allows for the extraction of the underlying reaction rate constants (Figure 2B). Notably, even if only one rate constant $k_{\mathrm{obs}}$ is experimentally observable, a nonlinear variation of $k_{\text {obs }}$ with ligand concentration is evidence for a multistep binding mechanism, and the exact dependence of $k_{\mathrm{obs}}$ on concentration gives information on its nature (Tummino and Copeland, 2008; Vogt and Di Cera, 2012). A shortcoming of these methods is that they do not offer site-specific resolution, and the exact nature of the event leading to a change in the observed signal is usually not known.

\section{NMR Spectroscopy}

NMR spectroscopy is a sensitive method to investigate binding reactions in proteins and can yield residue-specific information on binding sites, affinities and mechanisms (Zuiderweg, 2002). Typically, binding is monitored by observing the NMR resonances of an isotope- $\left({ }^{15} \mathrm{~N}\right.$ - and/or ${ }^{13} \mathrm{C}$ - $)$ labeled protein in the presence of unlabeled, i.e., NMR-inactive ligand. Ligand binding induces changes in the magnetic environment of spins in the residues involved in the interaction, reflected in changes in the chemical shifts of NMR resonances and/or their line shapes. Importantly, the underlying dynamics of the exchange of a spin between free and bound states affect NMR observables and can be measured using suitable experiments (Mittermaier and Kay, 2009). Thus, although NMR is an equilibrium technique, it can nevertheless provide access to kinetic details of a binding reaction and thereby its mechanism.

How NMR chemical shifts are affected by titrating with increasing amounts of ligand depends on the underlying exchange rate $k_{\text {ex }}$ between free and bound state, which, for the simple two-state binding scheme of Equation (1), is equal to the $k_{\text {obs }}$ observed in kinetic experiments $\left(k_{\text {on }}[\mathrm{B}]+k_{\text {off }}\right.$ for the exchange rate relevant if molecule $\mathrm{A}$ is observed). In the so-called fast exchange regime, i.e., if $k_{\mathrm{ex}}$ is much larger than the chemical shift difference between free- and bound-state signals $\left(k_{\mathrm{ex}} \gg \Delta \omega\right)$, a single resonance peak is observed for a given spin which moves from the free-state to the boundstate position in the course of the titration. Conversely, for slow exchange $\left(k_{\text {ex }} \ll \Delta \omega\right)$, the free-state resonances disappear upon titration, while the bound-state signals progressively appear in the spectrum (Figure $2 \mathrm{C}$ ). For intermediate exchange $\left(k_{\mathrm{ex}} \approx\right.$ $\Delta \omega)$, a combination of the two phenomena may be observed, but usually strong broadening or even disappearance of resonance signals occurs (Mittermaier and Kay, 2009). Notably, such titration experiments can already point to the presence of more complex binding mechanisms via, for example, peaks following a curved path (Arai et al., 2012), or combinations of fast- and slowexchange behavior in individual resonances (Sugase et al., 2007a). Peaks that remain exchange-broadened even in the presence of excess ligand have also been observed in IDP interactions with their partners (Jensen et al., 2011; Schneider et al., 2015), suggesting that the interaction kinetics are characterized by more than two states, for example if the bound state exhibits additional dynamics on the $\mu \mathrm{s}-\mathrm{ms}$ timescale.

On the timescales typical for protein-protein binding ( $\mu s-\mathrm{ms}$ range), NMR experiments useful for characterizing the exchange underlying such spectral changes are rotating-frame $\left(\mathrm{R}_{1 \rho}\right.$; Palmer and Massi, 2006) and Carr-Purcell-Meiboom-Gill (CPMG) relaxation dispersion (Palmer et al., 2001) as well as chemical exchange saturation transfer (CEST) experiments (Vallurupalli et al., 2012). In the context of analyzing IDP binding interactions, especially CPMG relaxation dispersion has been used. This experiment is normally conducted under conditions where either the free or the bound state of the protein under study is dominant and the respective other state(s) spectrally invisible due to its/their low population and preferential exchange broadening. If the exchange between free and bound states occurs on a timescale between about 100 and $2500 \mathrm{~s}^{-1}$, its contribution to the effective transverse relaxation rate $R_{2, \text { eff }}$ (and thus the linewidth) of the visible NMR signals can be quenched by the application of a train of $180^{\circ}$ pulses of increasing frequency $\nu_{\text {CPMG }}$ (Mittermaier and Kay, 2009). Fitting the resultant dependence of $R_{2 \text {,eff }}$ on $\nu_{\text {CPMG }}$ to a model of the underlying exchange yields its exchange rate(s) $k_{\mathrm{ex}}$, the populations of the states involved, and the chemical shift differences between them (Figure 2D). Individual rate constants $k_{\text {on }}$ and $k_{\text {off }}$ may be extracted from $k_{\text {ex }}$ by measuring relaxation dispersions at different concentrations or temperatures, or by knowledge of the dissociation constant, $K_{\mathrm{d}}$, of the interaction. In practice, the complexity of systems that can usefully be addressed using this strategy is limited to three exchanging states. NMR 

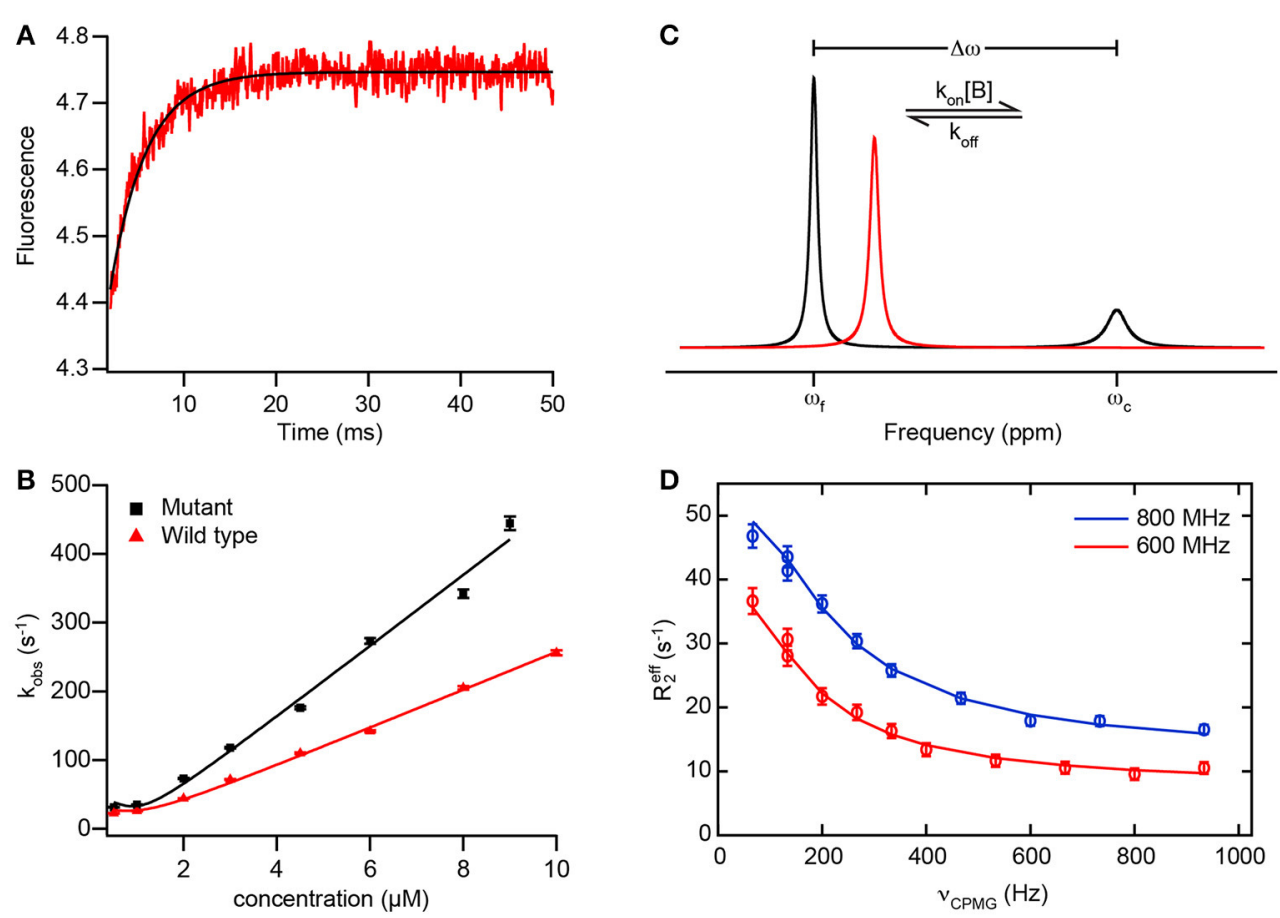

FIGURE 2 | Example data from transient kinetics and NMR to analyze binding mechanisms. (A) Fluorescence trace of a binding reaction measured in a stopped-flow experiment. The fluorescence of an introduced tryptophan residue (W2108) of NCBD(Y2108W) is monitored upon binding of disordered ACTR (see text for details). Red, experimental data; black, fit of an exponential function to obtain $k_{\text {obs }}$. (B) Dependence of the rate constant $k_{\text {obs }}$ observed in experiments as depicted in (A) with varying concentrations of wild-type ACTR (red) and a mutant ACTR variant with increased helix propensity (black). Solid lines are fits using the general equation for reversible association of two molecules (valid also for non-pseudo-first order conditions; Malatesta, 2005). (A,B) adapted from lešmantavičius et al. (2014) with permission of John Wiley \& Sons, Inc. (C) Schematic 1D NMR spectra of a spin undergoing exchange between two states with different chemical shifts, such as in a reversible binding interaction as in Equation (1). The spin is assumed to be in molecule A, such that the effective association rate constant is given by kon[B]. The free state (chemical shift $\omega_{\mathrm{f}}$ ) is assumed to be dominant and the complex (chemical shift $\omega_{\mathrm{c}}$ ) a minor state. Black, spectrum for intermediate to slow exchange $(k$ ex $<$ $\Delta \omega)$ with two resolved resonance lines for free and bound state; red, spectrum for intermediate to fast exchange $\left(k_{e x}>\Delta \omega\right)$ with one averaged resonance signal. In both cases, the effective transverse relaxation rate (and thus the linewidth) of the signals contains a contribution from the exchange, leading to additional line broadening. Note that the minor signal in the black spectrum is preferentially broadened due to its larger exchange contribution $k_{\text {off }}$, which can lead to broadening beyond detection. (D) Example data from a CPMG relaxation dispersion experiment measured at two static magnetic fields (red, $600 \mathrm{MHz}$, blue, $800 \mathrm{MHz}{ }^{1} \mathrm{H} \mathrm{Larmor}$ frequency) on the carbonyl ${ }^{13} \mathrm{C}$ of residue 475 in the PX binding site of Sendai virus $\mathrm{N}_{\text {TAlL }}$ in the presence of $8 \%$ (molar) of PX (see text for details; Schneider et al.,

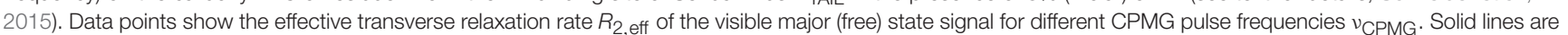
fits to the data using a model of exchange between three states, corresponding to free $\mathrm{N}_{\mathrm{TAlL}}$, encounter and final complex, allowing for extraction of the rate constants, populations, and chemical shift differences along the interaction trajectory.

signals are broadened by exchange on a similar timescale as the one CPMG experiments are sensitive to ( $\mu s-\mathrm{ms})$; consequently, line broadening may hamper data analysis.

\section{Molecular Simulations}

Simulations can, in principle, visualize biomolecular function and interactions in atomic detail and thus allow to elucidate the underlying mechanisms that are often difficult to access from experimental data alone. Recent years have seen a steady increase in both computing power and availability of resources for simulation techniques, from in-house clusters and graphical processing unit- (GPU-) based algorithms to large-scale computing facilities. However, both the models and parameters used as well as the simulation results need to be calibrated against and verified by experimental data to ensure that meaningful results are obtained. IDP systems pose some particular challenges for simulations. Due to their conformational heterogeneity, extensive sampling is required to ensure that the conformational space is adequately covered by the simulation. In addition, compared to their large number of degrees of freedom, experimental data on IDPs is typically sparse, i.e., their characterization is an underdetermined problem and care must be taken, e.g., by cross-validation approaches, to avoid overfitting. Finally, the accuracy of modern force fields for IDP simulations is not well characterized, and standard combinations of force fields and water models primarily developed for folded proteins may not be appropriate for simulations of IDP systems.

Standard molecular dynamics (MD) simulations of IDPs, using all-atom or united-atom representations (the latter with most nonpolar hydrogen atoms "collapsed" into the heavy atom to which they are bound), typically require long simulation times on the microsecond timescale to yield reasonable agreement with experimental data (Lindorff-Larsen et al., 2012). Most commonly, variants of the quantum mechanics-based AMBER 
or the empirically parametrized CHARMM and GROMOS force fields are used. Some variants have been optimized against NMR parameters, such as AMBER ff99SB-ILDN (LindorffLarsen et al., 2010) or CHARMM22* (Best and Hummer, 2009), or improved directly using NMR observables, like AMBER ff99SBNMR1-ILDN, which also contains improved representations of torsion angle potentials ( $\mathrm{Li}$ and Brüschweiler, 2010). Insufficient solvation of proteins has been observed using current force fields, which is particularly problematic for IDPs (Mercadante et al., 2015). To address this problem, short-range protein-water interactions can be modified in current water models such as TIP3P or SPC known to accurately reproduce the structure of liquid water (Horn et al., 2004; Wang L.-P et al., 2014; Henriques et al., 2015); alternatively, a new water model, TIP4P$\mathrm{D}$, has been proposed to correct for underestimation of London dispersion interactions (Piana et al., 2015). Extensive simulations on disordered peptides and validation against experimental NMR and small-angle X-ray scattering (SAXS) data have indicated that CHARM22* in conjunction with the TIP4P-D water model reproduces experimental data well, with the notable exception of NMR residual dipolar couplings (RDCs; Rauscher et al., 2015). This shortcoming has been observed for all available force fields and is likely due to the sensitivity of RDCs to both local and longrange structuration. Correspondingly, a bias in current force fields toward overly compact structures-as measured by, e.g., radius of gyration-has been observed (Henriques et al., 2015), which may partly explain why these force fields perform well for compact, folded proteins, but less so for simulations of IDPs where correct reproduction of their flexible and extended nature is essential. The TIP4P-D water model has also been observed to overly destabilize transient secondary structure (Salvi et al., 2016). Efforts have been made to correct for these deficiencies, with the specific aim to better reproduce IDP conformational sampling, for example by corrections to the dihedral potentials of disorder-promoting residues in AMBER ff99SB-ILDN, with some improvement in reproduction of NMR chemical shifts (Wang W. et al., 2014; Ye et al., 2015). Nevertheless, it appears that current force fields and water models are still not calibrated accurately enough to fully capture conformational sampling in IDPs, especially in the presence of transient structure, warranting caution in the interpretation of IDP simulations in terms of underlying mechanisms.

Coupled folding and binding of IDPs to their partners is typically still out of reach of classical MD simulations due to the large number of degrees of freedom as well as the extensive conformational transitions involved. To overcome the sampling limit of classical $\mathrm{MD}$, different approaches can be used. Coarse-grained (CG) simulations reduce the number of degrees of freedom by simplified representations of the molecular system, parametrizing individual amino acids using only one or a few hard spheres (Takada et al., 2015). Compared to all-atom simulations, this yields better agreement of the radius of gyration of IDPs with experiment (Smith et al., 2014). CG simulations are often combined with empirical "Gō-type" potentials to simulate protein-protein binding (Ueda et al., 1978; Karanicolas and Brooks, 2002). Here, terms for non-bonded interactions can be included which favor contacts between those residue pairs that are in contact in a known native structure of the complex investigated (De Sancho and Best, 2012). For protein binding or unfolding, Monte Carlo (MC) simulations have also been used. Here, the properties of the system are computed by repeated random sampling whose outcome is periodically accepted or rejected on the basis of a deterministic principle, i.e., among the conformations of a molecule corresponding to two consecutive steps, the one with minimal energy is chosen (Metropolis criterion; Irbäck and Mohanty, 2006; Staneva et al., 2012).

On the other hand, various enhanced sampling techniques for MD simulations have been proposed. One of the most widely used methods is parallel tempering or replica exchange molecular dynamics (REMD; Okamoto, 2004; Tai, 2004; Ostermeir and Zacharias, 2013). Here, several copies (replicas) of the system are simulated independently and simultaneously at different simulation temperatures. At preset intervals, pairs of replicas are exchanged with a specified transition probability. This allows the system to escape from being trapped in locally stable states at low simulation temperature, while preserving the canonical distribution of sampled states within each replica. Instead of using the simulation temperature as replica coordinate (T-REMD), it is also possible to vary the force field or system Hamiltonian across replicas. While improving sampling, REMD is computationally expensive and can become prohibitive for large proteins. An alternative approach known as metadynamics is based on the dynamics performed by a few collective variables of the atomic coordinates (Laio and Parrinello, 2002). In a metadynamics simulation, the system is driven not only by its potential energy, but also by a biasing history-dependent potential constructed as a sum of user-defined Gaussians centered along the trajectory of the collective variables which "fill" the free-energy surface to drive the system away from states that were already visited. Two variants of this technique have mostly been used: well-tempered metadynamics (W-META), a variant that adds an adaptive bias along the trajectory of the collective variables by varying the Gaussian height (Barducci et al., 2008); and bias exchange metadynamics (BE-META), which combines standard metadynamics with the replica exchange approach, allowing for extensive sampling of the free energy landscape (Domene et al., 2015). Recent studies have demonstrated that both BE-META and T-REMD are capable of reproducing secondary structure and lowly populated conformations of disordered peptides (Do et al., 2014; Zerze et al., 2015).

\section{POSSIBLE ADVANTAGES OF INTRINSIC DISORDER}

The possible consequences and advantages of intrinsic disorder for protein function and interactions have been extensively discussed (Dunker et al., 2002; Tompa, 2002; Dyson and Wright, 2005; Wright and Dyson, 2015). It appears that the specific qualities of disorder are well suited for the functions in interaction and regulation frequently fulfilled by IDPs. For example, their extended, flexible nature may be advantageous for 
the assembly of complexes due to facile access to the binding sites on an IDP and lack of steric hindrance (Tompa, 2002). Similarly, disordered regions can easily be accessed by modifying enzymes such as kinases, which may be why posttranslational modification sites are also often found in disordered regions (Iakoucheva et al., 2004). At the same time, disordered proteins can form extended binding interfaces that are large compared to their own size, offering the possibility of specific, high-affinity partner recognition (Mészáros et al., 2007; Dogan et al., 2014). On the other hand, their plasticity allows them to bind to different partners, using different or even identical binding sites, a phenomenon known as promiscuity (Tompa et al., 2005; Oldfield et al., 2008). Furthermore, turnover of IDPs in the cell is usually more rapid due to their susceptibility to proteases, which may be of advantage in signaling (Wright and Dyson, 1999; Dyson and Wright, 2005).

Two possible advantages of intrinsic disorder for proteinprotein interactions have been put forward and discussed particularly frequently: an increased association rate $\left(k_{\text {on }}\right)$ due to a "fly-casting" effect (Shoemaker et al., 2000), and an ability to achieve high binding specificity without concomitant high affinity (Zhou, 2012). We will look at these two propositions in more detail in the following sections.

\section{High Association Rates: Fly Casting vs. Pre-formed Structure}

The original proposition of the "fly-casting" effect stated that an unfolded protein should be able to form initial interactions with its binding partner already at a greater distance than a folded protein, leading to an increased capture radius and a "reeling in" of the partner to its binding site on the IDP (or vice versa; Shoemaker et al., 2000). This was predicted to lead to an, actually relatively modest, increase in the association rate by a factor of 1.6; however, larger rate enhancements have been suggested for other, more realistic models than were used in the original derivation (Shoemaker et al., 2000; Zhou et al., 2012). Experimentally, very fast association rates around the estimated diffusion limit for folded proteins (up to about $10^{9}-10^{10} \mathrm{M}^{-1} \mathrm{~s}^{-1}$ ) are found surprisingly frequently for IDPs (Sugase et al., 2007b; Arai et al., 2012; Shammas et al., 2013; Dogan et al., 2015; Milles et al., 2015). Association rates exceeding $10^{7} \mathrm{M}^{-1} \mathrm{~s}^{-1}$ are invariably associated with electrostatic attraction (Zhou and Bates, 2013). Indeed, IDPs in general tend to exhibit an elevated net charge (Uversky, 2002), and the vicinities of IDP binding sites have been described to be enriched in complementary charges (Ganguly et al., 2012). However, even if corrections for the effects of electrostatic attraction are applied, $k_{\text {on }}$ values in the diffusion-limited range have been found for IDPs $\left(10^{5}-10^{6}\right.$ $\mathrm{M}^{-1} \mathrm{~s}^{-1}$ ) (Shammas et al., 2013; Dogan et al., 2015; Milles et al., 2015). This suggests that disorder per se may indeed speed up the association process, via fly casting or otherwise. However, the number of kinetic studies of IDP association reactions is still relatively limited, and overall, a wide range of association rate constants has been measured for IDPs, similar to what has been observed for folded proteins (Dogan et al., 2014). Thus, so far it does not seem warranted to claim that IDPs can generally achieve faster association than folded proteins.

Fly casting is difficult to prove or disprove experimentally, largely owing to the difficulty of changing the level of disorder in one of the partners of a binding interaction without affecting other factors as well. Note that the original publication considered a comparison between a completely and a partly folded protein to derive the prediction of a 1.6-fold increase in $k_{\text {on }}$. However, most experimental assessments which can be related to the fly-casting hypothesis have rather involved short binding sites of IDPs exhibiting partial order, such as a transient helix, and modified the propensity to form such structure via mutations or cosolvents. In most of these experiments, stabilizing (secondary) structure was however found to increase association rates, albeit to different extent (Rogers et al., 2013, 2014b; Iešmantavičius et al., 2014; Arai et al., 2015), contradicting the fly-casting hypothesis. The association of the p27 cyclindependent kinase (CDK) inhibitor with the cyclin A-Cdk2 complex appears to be an exception; here, stabilizing the linker helix slowed down the time course of inhibition (Bienkiewicz et al., 2002). However, the mutations employed also changed the charge of p27. Moreover, the linker helix makes only few contacts with the cyclin A-Cdk2 complex and likely functions mostly as a spacer between the cyclin A- and Cdk2-binding domains of p27 (Lacy et al., 2004). Investigations of the kinetics of different proteins, with different degrees of disorder, binding to a single target have suggested no influence of pre-formed structure on association rates, or rather kinetic advantages for the more disordered proteins (Shammas et al., 2014; Dogan et al., 2015); however, comparing different proteins with regard to an individual quality is inherently difficult. Thus, while the experimental evidence is not entirely conclusive in this regard, it appears nevertheless that stabilization of the bound-state structure in an IDP prior to interaction leads to faster association rates.

CG simulations with a Gö-like potential of the interaction of the phosphorylated kinase inducible domain ( $\mathrm{pKID}$ ) of the cAMP response element binding protein (CREB) with the KIX domain of the CREB-binding protein (CBP) have also suggested that the kinetic advantage of an increased capture radius would be offset by the slower translational diffusion of a disordered protein (Huang and Liu, 2009). This likely only applies to large-scale changes in disorder, and thus diffusion coefficient, envisioned in the original fly-casting hypothesis; stabilization of a transient helix in the binding site of an IDP was not observed to have an effect on its diffusion (Iešmantavičius et al., 2014). However, based on these simulations, another mechanism was proposed by which disorder could speed up protein-protein association, namely a reduction of the free-energy barrier between initial and final complex due to facilitated folding after initial binding (i.e., induced fit, see also below), or equivalently, an increase in the number of collisions leading to productive binding (Huang and Liu, 2009). The expected increase in $k_{\text {on }}$ from this effect is again rather modest, a factor of 2.5 (Huang and Liu, 2009). Similarly, in Gō model simulations on the interaction of the C-TAD domain of hypoxia-inducible factor- $1 \alpha$ (HIF $1 \alpha)$ to the TAZ1 domain of CBP, the observed association rate approached 
the fast experimental one only if the model allowed for nonnative contacts to occur, leading to a broad distribution of encounter complexes productive for binding (De Sancho and Best, 2012). Electrostatic interactions appear to play a crucial role in this process by reducing the redissociation rate after initial encounter, as well as by increasing the probability of nativelike topologies in the collision complexes (Ganguly et al., 2013). A facilitated transition between initial and final complex bears some resemblance to a fly-casting mechanism, albeit without an effect of a larger capture radius. Notably, rather than increasing association rates beyond those achievable by ordered proteins, this effect of disorder seems to avoid the orientational restraints and steric hindrance that would result if IDPs had to rigidly dock their binding partners, at least in those cases where the binding interface is more complex and extended (Zhou et al., 2012). In the aforementioned case of p27 binding to the cyclin A-Cdk2 complex, it was noted that, due to steric clashes, p27 could not bind if it were rigid in its bound conformation; this would explain the requirement for a flexible linker helix found experimentally (Bienkiewicz et al., 2002; Zhou et al., 2012). One could thus argue that the flexibility of IDPs counterbalances the negative effects on association rates that their often extended binding interfaces would otherwise have, globally leading to a similar range of association rates as found in folded proteins (Dogan et al., 2014).

Regardless of the effects of pre-formed structure on kinetics, stabilization of bound-state secondary structure has always been observed to increase affinity and lead to corresponding changes in downstream functional responses of IDPs (Parker et al., 1998, 1999; Petros et al., 2000; Rogers et al., 2014b). For example, stabilizing the transient helix with which the transactivation domain (TAD) of the tumor suppressor p53 binds to the repressor oncoprotein MDM2 leads to stronger binding and concomitant effects on target gene expression and cell cycle regulation (Zondlo et al., 2006; Li et al., 2010; Borcherds et al., 2014). In the disordered domain D2 of hepatitis C virus (HCV) protein NS5A, a short structural motif dubbed the PW turn was identified whose disruption abolished HCV replication and affected interaction with the host protein cyclophilin A (Dujardin et al., 2015). These and other examples underline the importance of pre-formed structure in IDP binding and indicate that it typically has a functional role.

\section{Affinity and Specificity}

It has been suggested that the loss of conformational entropy of IDPs associated with folding upon binding results in an overall unfavorable entropic contribution to binding, thereby uncoupling binding strength from specificity, i.e., allowing for highly specific binding without high affinity (Tompa, 2002; Fuxreiter et al., 2004; Dyson and Wright, 2005; Zhou, 2012). This has been cited as an example of enthalpy-entropy compensation (Fuxreiter et al., 2004) and as an advantage in the context of signaling, where interactions have to be reversible to assure that a signal can be switched off again. However, it is not entirely clear how disorder can uncouple specificity and affinity, since the two are intimately linked by definition. Thermodynamic specificity, in essence, consists in differences in (relative) affinity to multiple possible targets and therefore also depends on the availability and concentration of binding partners (Zondlo et al., 2006). Thus, an interaction of weak or moderate affinity may well be highly specific, however only as long as any possible competing interaction exhibits even weaker affinity (or the concentrations of other binding partners are sufficiently low). Also, in principle, if a binding interaction is accompanied by a large unfavorable entropy change, and if enthalpy-entropy compensation is at play, the result should not be an overall low affinity of the complex, but rather a concomitant large favorable enthalpic contribution, as has been noted in the context of folding upon binding in protein-DNA interactions (Spolar and Record, 1994). Finally, IDPs also engage in promiscuous interactions with different binding partners, even via identical binding sites as in the case of the C-terminal regulatory region of p53 (Oldfield et al., 2008), possibly leading to different functional responses (moonlighting; Tompa et al., 2005). It is not clear how intrinsic disorder would be able to both assure high binding specificity and still permit promiscuous interactions.

In fact, the reduction in entropy associated with folding upon binding of an IDP does not generally entail an overall unfavorable entropic contribution to binding. First, pre-formed structure corresponding to the bound conformation, as well as disordered segments not directly involved in the binding, reduce the entropy penalty of folding upon binding (Fuxreiter et al., 2004), as is the case for so-called "fuzzy complexes" that retain dynamics even within the binding regions (Mittag et al., 2008; Tompa and Fuxreiter, 2008). But even for IDP complexes reaching a stable bound conformation, the gain in solvent entropy from the release of solvation water molecules can be considerable, especially given that IDPs tend to form comparatively large, extended binding interfaces with pronounced hydrophobic character (Dyson and Wright, 2005; Mészáros et al., 2007; Ganguly et al., 2012; Dogan et al., 2014). The overall entropy of folding and binding of an IDP to its partner may thus well be favorable, as, for example, in the case of the interaction of the disordered transactivation domain of the transcriptional activator c-Myb with the KIX domain of CBP (Parker et al., 1999). Notably, the formation of a large hydrophobic interface typically also leads to a favorable binding enthalpy, which can compensate for unfavorable entropic contributions, as seen for example in the interaction of the disordered activation domain of the activator for thyroid hormone and retinoid receptors (ACTR) with the nuclear coactivator binding domain (NCBD) of CBP (Demarest et al., 2002).

Overall, as is the case for association rates, IDP interactions exhibit a wide range of affinities, including very high ones, as do folded proteins (Dogan et al., 2014). Notably, nanomolar dissociation constants have been found even for IDP complexes involved in signaling, such as ACTR-NCBD (Demarest et al., 2002), p27-cyclin A-Cdk2 (Lacy et al., 2004), or the complex between disordered PUMA and folded Mcl-1 from the mammalian Bcl-2 family of apoptosis regulators (Rogers et al., $2014 b$ ). How the signal associated with the formation of such complexes is switched off again, given their high affinity, is not clear. However, other, competing interactions of comparable affinity may be involved. As mentioned, specificity essentially corresponds to relative affinity and thus depends on the context 
in which a particular complex exists. In any case, intrinsic disorder does not seem to generally lead to low-affinity and/or high-specificity complexes.

\section{CONFORMATIONAL SELECTION, INDUCED FIT AND BEYOND}

\section{Distinguishing Conformational Selection and Induced Fit}

The discussion about the mechanisms used by IDPs to bind to their partners has often been framed in the context of a supposed dichotomy of conformational selection vs. induced fit (Figure 1), while more recent results tend to suggest that IDP binding mechanisms may be more complex, exhibiting various mixed, intermediate mechanisms, or a coexistence of these two limiting cases (Espinoza-Fonseca, 2009; Csermely et al., 2010). Conformational selection, in a strict sense, implies that the bound-state conformation of an IDP preexists in its free state and that exclusively this conformation is binding competent. Induced fit in the context of IDPs, on the other hand, entails that binding occurs in a more or less unfolded state and that conformational rearrangements to the final bound state take place in a unimolecular reaction within the complex. Evidence for both mechanisms has been found in binding of various IDPs to their partners, sometimes even for a single IDP (Gianni et al., 2012; Arai et al., 2015), but conclusive proof of one or the other is usually difficult and may not reflect the true complexity of the underlying binding mechanism.

As mentioned, a necessary condition for a conformational selection mechanism is that molecules assuming the bound conformation preexist in the free state ensemble of the IDP. NMR and MD studies have often found evidence for such preformed conformers closely resembling the bound state, for IDPs as well as for folded proteins, suggesting that conformational selection plays a role in their binding (Lange et al., 2008; Boehr et al., 2009; Kjaergaard et al., 2010; Jensen et al., 2011; Krieger et al., 2014). However, pre-formed bound-state structure per $s e$ is not evidence for a conformational selection mechanism, since its existence does not prove its (exclusive) implication in binding (Dogan and Jemth, 2014). Such evidence normally requires kinetic measurements of the reaction rate constants over a range of concentrations of at least one of the binding partners. However, in many cases, multistep reactions such as folding before or after binding do not become directly evident as, for example, multiexponential relaxation in non-equilibrium experiments or evident three-state behavior in NMR relaxation dispersion data.

For transient kinetics experiments, even if only a single $k_{\text {obs }}$ is observable experimentally, a multistep reaction can nevertheless become apparent if $k_{\mathrm{obs}}$ varies nonlinearly with the concentration of the binding partner. In that case, a $k_{\mathrm{obs}}$ increasing or decreasing hyperbolically with concentration has often been taken as evidence for an induced fit or conformational selection mechanism, respectively (Tummino and Copeland, 2008). However, this is only valid under the so-called rapid equilibrium assumption, where binding is assumed to be fast relative to the folding step (Vogt and Di Cera, 2012). This assumption is not necessarily justified, especially in the context of IDPs forming short elements of secondary structure, which can occur on a sub- $\mu$ s timescale (Eaton et al., 1997). Consequently, it has been shown that, while a $k_{\text {obs }}$ decreasing with binding partner concentration can only be explained by conformational selection, a hyperbolic increase in $k_{\text {obs }}$, as is often observed experimentally, can actually be accounted for by both induced fit and conformational selection mechanisms, leading to the suggestion that binding by conformational selection may be much more widespread than previously thought (Vogt and Di Cera, 2012, 2013). Recently, it has been pointed out that a distinction between the two binding mechanisms may be made in kinetic experiments by varying the concentrations of both reaction partners in separate experiments (Gianni et al., 2014). Induced fit yields a hyperbolically increasing $k_{\mathrm{obs}}$ in both cases. Conformational selection, on the other hand, results in a linear $k_{\text {obs }}$ variation if the concentration of the species undergoing conformational change is varied, while hyperbolic behavior (increasing or decreasing $k_{\mathrm{obs}}$ ) is retained for variation of the concentration of the other partner. In practice, it may however be difficult to obtain data for a wide enough range of protein concentrations to draw clear mechanistic conclusions. Alternatively, binding kinetics may be studied at different temperatures or with different ligands; however, the latter method assumes that only the microscopic rate constants, not the basic mechanism, would change for different ligands (Vogt and Di Cera, 2012).

\section{Conundrums in Experimental Data}

Kinetic data that can satisfactorily be explained by conformational selection or induced fit alone have been described in the literature, often for enzymes binding to their substrates (Kirschner et al., 1966; Wong et al., 1991; Kim et al., 2007; Johnson, 2008). Frequently, however, NMR or non-equilibrium kinetics measurements of IDP binding interactions give no direct indications of multistep mechanisms at all, with NMR relaxation dispersion data being well fit by two-state models and $k_{\text {obs }}$ from rapid mixing or temperature jump experiments varying linearly with ligand concentration (Shammas et al., 2013; Rogers et al., 2014b; Arai et al., 2015). Nevertheless, since both folding and binding take place, the underlying mechanism should be more complex than two-state. One of the processes involved may simply be too fast to be observed or be associated with too small a change in measurement parameters (fluorescence or NMR chemical shift, for example). Indeed, apparent two-state behavior is frequently observed in IDPs with simple bound topologies such as a single stretch of helix. In these cases, information on the binding mechanism and the role of pre-formed structure is often sought by modifying secondary structure content via mutation or by adding (secondary) structure stabilizing compounds such as trifluoroethanol (TFE; Gianni et al., 2012; Rogers et al., 2014b).

As discussed above, stabilizing the-in most studies, helicalbound-state secondary structure in IDPs has always been observed to stabilize the complexes with their binding partners, i.e., to increase affinity, while the effect of increased secondary structure on the on rate $k_{\text {on }}$ of IDP binding is more ambiguous 
(Bienkiewicz et al., 2002; Gianni et al., 2012; Iešmantavičius et al., 2014; Rogers et al., 2014b; Arai et al., 2015). Nevertheless, in a majority of cases, $k_{\text {on }}$ increases with increasing content of bound-state-like secondary structure within IDP binding sites, although to different extents. This behavior would be expected in a conformational selection scenario; increasing the population of the binding-competent conformer should lead to faster association. However, an increase in $k_{\text {on }}$ strictly is only evidence for a reduced free energy of the rate-limiting transition state of the reaction with respect to the free state. This ratelimiting step may well correspond to folding occurring after initial binding; in that case, binding would be accelerated by the increased propensity of the binding site for the boundstate structure, rather than by increased availability of an obligatory pre-folded conformation (Iešmantavičius et al., 2014). Conversely, the observed complex stabilization by increased freestate secondary structure content has typically been traced to a reduction in the off rate $\left(k_{\text {off }}\right)$, i.e., a lower free energy of the final bound state with respect to the rate-limiting transition state (Gianni et al., 2012; Rogers et al., 2014a,b). Such an effect is normally interpreted as folding after binding, i.e., an induced fit mechanism. Again, however, this depends on where on the reaction coordinate the transition state occurs; it may correspond to a rate-limiting folding step that precedes binding. However, for binding reactions, it seems reasonable to assume that the transition state is already a complexed state; especially the formation of short segments of helical secondary structure in the free state of an IDP is unlikely to account for the rate-limiting step of a folding and binding interaction. Thus, if (de)stabilization of bound-state secondary structure affects $k_{\text {off }}$, a contribution of an induced-fit mechanism to the binding can be assumed.

Similar to $\Phi$ value analysis that relates activation free energy to equilibrium free energy of binding (Fersht and Sato, 2004), information on the transition state of a folding and binding reaction can be obtained from how $K_{\mathrm{d}}$ varies with $k_{\text {on }}$ or $k_{\text {off }}$ for a series of modifying conditions (such as mutations or addition of TFE or denaturant; Prakash, 2011). If, for a given molecular system, a Brønsted plot of $\log \left(k_{\text {on }}\right)$ or $\log \left(k_{\text {off }}\right)$ against $\log \left(K_{\mathrm{d}}\right)$ is linear (i.e., gives a linear free-energy relationship, LFER), the position of the transition state on the reaction coordinate can be estimated from the slope of the plot. A large negative slope (approaching -1) in a plot of $\log \left(k_{\mathrm{on}}\right)$ against $\log \left(K_{\mathrm{d}}\right)$ indicates a "late" transition state with the property investigated by the modifications (such as secondary structure or tertiary contacts) largely formed, analogously to large values in $\Phi$ value analysis. Note that the results of such studies depend on the type of modification made to the system investigated. For example, for the interaction of the transcription factor $\mathrm{c}-\mathrm{Myb}$ with the KIX domain of CBP, it has been postulated, based on kinetic binding studies in varying TFE concentrations, that $\mathrm{c}-\mathrm{Myb}$ is largely unstructured in the transition state (Gianni et al., 2012). However, based on mutagenesis, it was concluded that the transition state exhibits a high degree (about 89\%) of native order (Giri et al., 2013). It is difficult to envision how, in the transition state, the KIX binding region of $\mathrm{c}-\mathrm{Myb}$ could be mostly unfolded and nevertheless undergo such a large percentage of interactions characteristic of the final bound complex, where it is present in a helical conformation (Zor et al., 2004). However, the mutations used were not chosen to perturb a specific quality such as secondary structure or hydrophobic interactions, and they were situated both within and outside of the binding interface, making their relative effects on folding and binding difficult to estimate. In the $\mathrm{BH} 3$ motif of disordered PUMA that binds as a helix to Mcl-1, secondary structure content was more specifically targeted by mutating residues outside of the binding interface to proline or glycine (Rogers et al., 2014a,b). Here, a modest effect on $k_{\text {on }}$ (variation over one order of magnitude) was observed, but a much stronger one (up to 1000 -fold) on $k_{\text {off }}$, again interpreted as evidence for an early transition state and binding of PUMA in a mostly disordered conformation (Rogers et al., 2014a,b). These results indeed show that no particular part of the boundstate secondary structure of PUMA is strictly required for its binding. However, the free state of PUMA has been observed to exhibit only about $20 \%$ overall helicity; a transition state with little secondary structure is thus not yet evidence against conformational selection. Since the mutations employed did not vary helical content to a great extent, a small effect on $k_{\text {on }}$ is perhaps not surprising. Also, some mutations introduced charge changes, and proline mutations are strongly disruptive to hydrogen bonding and may introduce additional complications via cis-trans isomerization.

In a study on the folding and binding of ACTR to the NCBD domain of CBP, helical secondary structure in free ACTR was targeted by carefully designed mutations to noninterface residues, explicitly excluding mutations to proline or involving changes in charge (Iešmantavičius et al., 2014). Resultant effects on helicity were monitored using both CD and NMR spectroscopy, and binding kinetics of the mutants to NCBD were measured by stopped-flow fluorimetry. Here, a clear correlation of free- state helical content, which varied in the range of $20-70 \%$ in the different mutants, with both $k_{\text {on }}$ and $k_{\text {off }}$, which both varied 2 - to 2.5 -fold, was found. The plot of $\log \left(k_{\text {on }}\right)$ vs. $\log \left(K_{\mathrm{d}}\right)$ resulted in a slope of -0.47 , while in an earlier study, mutations affecting the hydrophobic binding interface yielded an average $\Phi$ value of 0.14 (Dogan et al., 2013). This has been interpreted as a transition state in which crucial hydrophobic intermolecular interactions are only formed to $14 \%$, but with the ACTR binding region already $47 \%$ helical (Iešmantavičius et al., 2014), indicating a mixed mechanism in which partly preformed conformers of ACTR bind and fold further to their final bound conformation within the complex. A reanalysis of the mutagenesis data performed in (Giri et al., 2013) in terms of the effect of the different mutations on helical secondary structure content also indicated that helicity is in fact correlated with $k_{\text {on }}$ in the interaction of c-Myb with KIX (Arai et al., 2015).

\section{Mixed Multistep Mechanisms: A Potential Solution}

Clearly, the different effects of mutations and cosolvents on folding and binding processes are often difficult to separate. However, an emerging consensus from this type of experiment seems to be that induced fit always has a role in the systems investigated, but that mixed mechanisms seem to be 
at play, as seen from the finding that pre-formed secondary structure affects on rates and is reflected in the degree of structuration of the transition state. It has already been noted that IDP binding mechanisms may be more complex than either conformational selection or induced fit, and that various combinations or intermediates of these two extremes need to be considered (Espinoza-Fonseca, 2009; Csermely et al., 2010). In particular, conformational selection and induced fit may coexist, with relative flux through the two pathways being determined by substrate concentration as well as the relative populations of the different forms of the protein undergoing a conformational change (Hammes et al., 2009). Reaction steps respectively exhibiting characteristics of conformational selection and induced fit may also occur sequentially in a multistep reaction (Espinoza-Fonseca, 2009; Csermely et al., 2010). Such complex behavior with parallel multistep pathways has been invoked in a detailed study for the folding and pyrophosphate binding of the Bacillus subtilis RNase $\mathrm{P}$ protein subunit (Daniels et al., 2014). Here, it was suggested that fast folding kinetics and low ligand concentrations favor conformational selection pathways, while slow folding and increasing ligand concentrations shift the balance toward induced fit pathways. With three conformational substates and two substrate binding sites, the system investigated in this study exhibits particular complexity; nevertheless, increasing evidence suggests that other IDPs may employ similar combinations of pathways and individual interaction steps in their interactions with binding partners.

As mentioned, an acceleration of binding by increased secondary structure content may reflect a rate-limiting folding step occurring after binding. However, there are indications for a role of partial conformational selection in such cases. As discussed above, very high, diffusion-limited association rates are recurrently found in interactions of IDPs with their partners, notably in the aforementioned PUMA-Mcl-1, cMybKIX, and ACTR-NCBD systems (Dogan et al., 2012; Rogers et al., 2013; Shammas et al., 2013). This is often cited as evidence for induced-fit type binding, since the requirement of a conformational selection mechanism for a pre-folded, possibly lowly populated, conformation should be incompatible with diffusion-limited association, where each encounter of the binding partners should lead to productive binding. However, it seems difficult to delineate the diffusion limit for disordered proteins, and even for induced-fit type binding, an energy barrier for folding after binding may remain that slows down the overall association (Rogers et al., 2013), likely in a similar way as conformational selection would. Most notably, however, the involvement of pre-formed structure in diffusion-limited interactions very likely depends on the degree of prestructuration of a particular molecule. For IDPs whose binding sites display a high content of secondary structure resembling the bound state, such as c-Myb(284-315) (70\%; Arai et al., 2015) or the disordered C-terminal $\mathrm{N}_{\text {TAIL }}$ region of the nucleoprotein from Sendai virus (up to 75\%; Jensen et al., 2008), it would likely be a pure inducedfit mechanism that would hamper fast association, given the prevalence of pre-formed conformers. It thus seems likely that, as described by Oas and coworkers for RNase P folding and binding
(Daniels et al., 2014), highly populated, fast-folding pre-formed structure would preferentially participate in binding, at least if the partner protein is not present in excess.

After initial binding of pre-folded conformers, further folding may still occur within the complex. Such behavior is likely when secondary structure is significantly populated in the free state of an IDP, but is extended further in the final bound state, as seen for example in binding of the $\mathrm{N}$-terminal region of the vesicular stomatitis virus (VSV) phosphoprotein $\mathrm{P}$ to the nucleoprotein N. Residues 25-31 of $\mathrm{P}$ form a transient helix populated up to $35 \%$ in the free state (Leyrat et al., 2012); in the $\mathrm{N}^{0} \mathrm{P}$ complex, $\mathrm{P}$ is helical in residues 17-31 (Leyrat et al., 2011). Similarly, in the transactivation domain of p53, residues 22-25 have been observed to populate helical secondary structure (Wells et al., 2008), while the crystal structure of the complex between this motif and the MDM2 oncoprotein features a helix in residues 19-25 (Kussie et al., 1996). Alternatively, the docking regions D1, D2, and D3 of the regulatory region of the mitogen-activated protein kinase (MAPK) kinase MKK7 all bind to the MAPK JNK1 in extended or polyproline conformation, despite sampling different regions of Ramachandran space in the free state (helical, polyproline, or random coil, respectively; Kragelj et al., 2015). For these interactions, an induced fit step appears obligatory since the bound-state conformation is not populated in the free ensemble, at least not to a measurable extent. Induced fit also certainly plays a role in the intriguing cases of individual disordered motifs binding to different partners in different conformations, as has been observed for residues 374-388 of the C-terminal regulatory region of $\mathrm{p} 53$ which bind to the proteins $S 100 \beta \beta$, sirtuin, CBP, and cyclin A2 in helix, sheet, or different coil conformations, respectively (Oldfield et al., 2008). Induced fit has indeed been observed in an MD study of the interaction of $S 100 \beta \beta$ with p53 and another disordered partner, the TRTK-12 fragment from the protein CapZ. Here, MD simulations were complemented with Monte Carlo calculations for improved sampling of the binding process. Despite their differing sequences and bound-state structures, both peptides were observed to employ similar induced-fit binding mechanisms via non-native encounter complexes formed in the periphery of the $S 100 \beta \beta$ binding pocket. Nevertheless, helical structure similar to the bound state was observed in simulations of the free peptides (Staneva et al., 2012). Gō-type coarse-grained simulations of other IDP interactions such as those of PUMA with Mcl-1 or HIF1 $\alpha$, p53, and ACTR with their cognate domains of CBP (TAZ1, TAZ2, and NCBD, respectively) have also found important induced fit contributions to the binding mechanisms, involving a broad range of encounter complexes. Here, significant folding typically occurred only after formation of at least $20 \%$ of native intermolecular contacts (De Sancho and Best, 2012; Ganguly et al., 2013; Rogers et al., 2014a).

NMR studies of IDP folding and binding support the notion of complex multistep interactions with different contributions of conformational selection and induced fit. A seminal study of Wright and coworkers on the interaction of the pKID domain of CREB with the KIX domain of CBP suggested a model of exchange between four states, namely the free proteins, a transient, nonspecific encounter complex, a partially folded 
intermediate, and the final complex (Sugase et al., 2007a). The latter three states were inferred from fits to relaxation dispersion data measured on the complex, while the initial step of formation of an encounter complex was indicated by resonance signals of free pKID shifting in fast exchange during titration experiments. Encounter complex and intermediate were found to be only partially structured, clearly showing that this interaction proceeds largely via an induced-fit mechanism. A different result was obtained for the binding to KIX of c-Myb, whose primary binding site on KIX is the same as for pKID. Here, NMR relaxation dispersion data were consistent with an effective twostate mechanism (Arai et al., 2015), as was the case for stoppedflow and temperature-jump kinetic experiments (Gianni et al., 2012; Giri et al., 2013; Shammas et al., 2013). Based on the latter, an induced-fit mechanism was postulated (Gianni et al., 2012; Giri et al., 2013). However, recent coarse-grained as well as allatom simulations of this interaction found no unique pathway for binding and indicated that c-Myb conformations with widely varying helical content can bind to KIX with similar probabilities (Ithuralde et al., 2016). In addition, in the transient kinetics experiments, a shorter $\mathrm{c}-\mathrm{Myb}$ construct exhibiting relatively low helical content in the free state (around 30\%) was used (Shammas et al., 2013). NMR data on the longer c-Myb (284-315) construct, on the other hand, rather indicated a fast free-state folding equilibrium. The large (70\%) helical content in this construct, the effect on $k_{\text {on }}$ of mutations affecting secondary structure, and the apparent high degree of structure in the transition state underline a role of conformational selection in this interaction (Giri et al., 2013; Arai et al., 2015). The differences apparent in the binding of pKID and c-Myb to KIX were related to their different roles in transcriptional activation, where largely unfolded KID requires induction by phosphorylation for high-affinity interaction with KIX, while the more helically preconfigured c-Myb acts as constitutive activator (Zor et al., 2002; Arai et al., 2015).

The previously mentioned case of the interaction of the ACTR activation domain with the NCBD domain of CBP has also been investigated by both non-equilibrium kinetics and NMR experiments, as well as Gō-type CG simulations. Here, an additional complication arises from the fact that both domains are disordered in their free states, however to different degrees. NCBD has been described as a molten globule (Kjaergaard et al., 2010), while ACTR is fully disordered with transient helical elements (Iešmantavičius et al., 2013). Both proteins thus undergo a coupled folding and binding reaction that has been described as mutual synergistic folding (Demarest et al., 2002). Free NCBD largely populates a conformation resembling its ACTR-bound state (Kjaergaard et al., 2010), while the H1 helix found in NCBD-bound ACTR is partly pre-formed in free ACTR to a population of $38 \%$ (Iešmantavičius et al., 2013). Transient kinetics experiments, as well as simulations, have suggested a multistep, largely induced-fit, binding mechanism (Dogan et al., 2012; Ganguly et al., 2013). However, as discussed above, the effects of mutations on binding kinetics have also indicated a partly helical transition state with few native tertiary interactions (Dogan et al., 2013) and a clear influence of helicity on association rate (Iešmantavičius et al., 2014). It thus seems likely that the pre-folded conformers present in the free-state ensembles of both
ACTR and NCBD play a role in the initial binding step. For example, as has been suggested, the interaction may be initiated by a pre-formed first helix of ACTR making weak native-like contacts with the first helix of NCBD (Dogan et al., 2013). Such a mechanism is supported by the respective timescales of folding and binding observed in this system. NMR line shapes indicate that formation of the (fluid) tertiary structure of NCBD occurs on a timescale of $10^{4} \mathrm{~s}^{-1}$, with transient helix formation in ACTR likely even faster (Eaton et al., 1997; Jensen et al., 2008), while the rate of complex formation is on the order of $500 \mathrm{~s}^{-1}$ or slower (Iešmantavičius et al., 2014). Pre-formed binding competent conformers of both proteins would thus be readily available for complex formation.

In another study relevant to the subject, we have investigated the interaction between disordered $\mathrm{N}_{\mathrm{TAIL}}$ and the PX domain of the phosphoprotein from Sendai virus $(\mathrm{SeV})$ using relaxation dispersion NMR experiments. The data were best explained by a three-state model in which one of the preexisting conformers of the highly helical free-state ensemble is initially stabilized in a nonspecific encounter complex with PX, as evidenced by the dominance of ${ }^{13} \mathrm{C}$ backbone chemical shift modulations during the first step of the interaction. From a helical periodicity in ${ }^{15} \mathrm{~N}$ and ${ }^{1} \mathrm{H}$ shifts that dominate the second step, we concluded that this $\mathrm{N}_{\text {TAIL }}$ helix then locks into its final bound position in a hydrophobic interhelical groove of $\mathrm{PX}$, at a rate coincident with intrinsic motion of that groove (Schneider et al., 2015; Figure 3). The first step of this model thus comprises both folding and binding which could not be resolved kinetically. However, the bound conformation is-with one residue difference-nearly identical to one of the helical conformers populated to $28 \%$ in the free state. In addition, the molar fractions of PX used $(<=15 \%)$ were below the population of that helical conformer, and the formation of helices in free $\mathrm{N}_{\text {TAIL }}$ is much faster-above $10^{5} \mathrm{~s}^{-1}$ from NMR data, in agreement with known helix folding rates (Eaton et al., 1997) - than the on rate, below $140 \mathrm{~s}^{-1}$ under the conditions measured. It can thus be assumed that, in these conditions, enough pre-folded $\mathrm{N}_{\text {TAIL }}$ conformers resembling the bound state will always be available for a conformationalselection type interaction in the first step of binding.

Interestingly, a temperature-jump kinetic study on the closely related $\mathrm{N}_{\text {TAIL }}-\mathrm{XD}$ system from measles virus $(\mathrm{MeV})$ has also found complex kinetics with at least two steps, but has come to the opposite conclusion that the interaction is characterized by a global induced-fit mechanism (Dosnon et al., 2015). This study used the kinetic test in which the concentrations of both binding partners are varied in separate experiments (Gianni et al., 2014) and found a hyperbolic dependence of the observed rate constant $k_{\mathrm{obs}}$ in both cases, in agreement with an induced-fit interaction. The sequence homology between the corresponding proteins in the two viruses is rather low (about $20 \%$ identity), so the mechanisms involved in binding may well be different. However, it is unlikely that even the temperature jump technique, appropriate for fast reactions, is sensitive to the very fast rate of helix formation and interconversion in the free $\mathrm{N}_{\text {TAIL }}$ proteins of both viruses (Eaton et al., 1997; Jensen et al., 2008, 2011). The curvature observed in the variation of $k_{\mathrm{obs}}$ with the concentrations of both $\mathrm{N}_{\mathrm{TAIL}}$ and XD from 


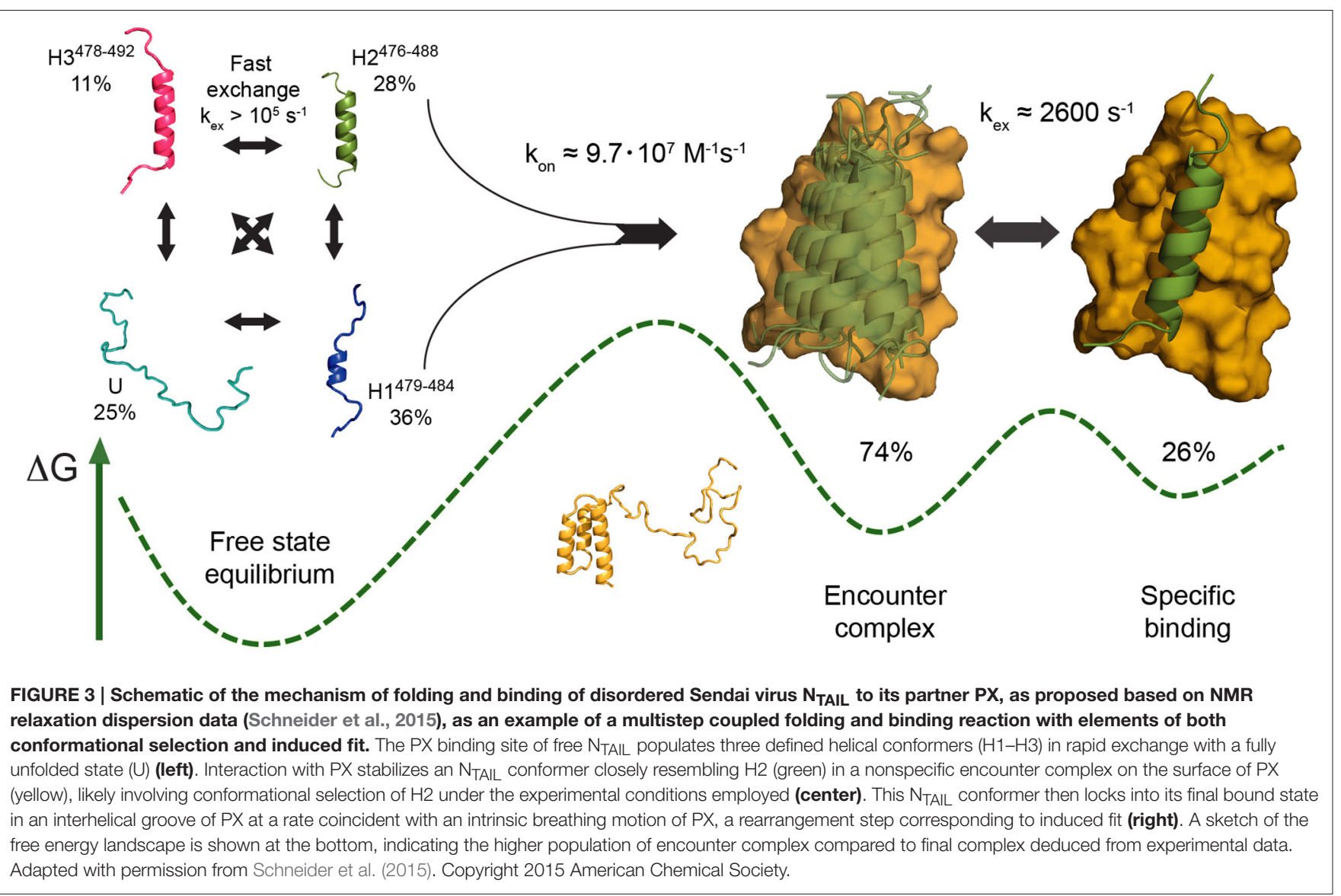

$\mathrm{MeV}$ confirms that a conformational change occurs within the complex between the two proteins. However, it is unknown what the observed change in tryptophan fluorescence corresponds to exactly at the molecular level. A "locking-in" of a pre-formed helical $\mathrm{N}_{\text {TAIL }}$ conformer on the surface of its binding partner, as inferred from NMR data on the SeV system, may also explain the unimolecular process detected in the temperature jump fluorimetry experiments on the $\mathrm{MeV}$ proteins. Thus, it appears likely that, in these two systems as well, conformational selectionand induced fit-type processes coexist in a sequential manner. The site-specific resolution offered by NMR spectroscopy clearly allows for additional detailed insight into the molecular processes underlying the measured kinetic parameters in these cases.

The $\mathrm{MeV} \mathrm{N}_{\mathrm{TAIL}}-\mathrm{XD}$ interaction has also been investigated by molecular simulations. Free $\mathrm{N}_{\text {TAIL }}$ was simulated using REMD, while simulation of the binding process either employed a hybrid potential, incorporating structural information on the bound state as attractive potential terms (Wang et al., 2013), or a combination of replica exchange and metadynamics (Han et al., 2016). Both studies reached similar conclusions about a sequential combination of conformational selection and induced fit in this system, in agreement with the experimental studies mentioned above. However, the composition of the simulated free-state ensemble in the study by Wang et al. differed somewhat from that of an ensemble based on experimental data (Jensen and Blackledge, 2014). In particular, experimental NMR RDCs were less well reproduced by the REMD ensemble, likely due to the prevalence of kinked helical conformers in the latter. The free-state ensemble described by Han et al. is similarly enriched in such structures, suggesting it is subject to the same issues regarding reproduction of experimental data. This observation underlines that current force fields still encounter problems in simulating the conformational sampling of IDPs in their isolated state, especially when transient structure is present. Consequently, caution should also be exercised in drawing conclusions on interaction mechanisms from molecular simulations.

\section{A Synthetic View of IDP Folding and Binding}

Overall, it appears that most coupled folding and binding reactions exhibited by IDPs are considerably more complex than could easily be described by either conformational selection or induced fit models. It has long been realized that these two mechanisms, while useful concepts and appropriate for the characterization of a range of known binding interactions, as well as individual reaction steps, are very likely too reductionist to describe the complexity of most biomolecular interactions entirely. The two limiting cases of flexible protein binding mechanisms seem to coexist not only as parallel pathways with 
flux-dependent relative importance (Hammes et al., 2009), but also sequentially in the multistep folding and binding reactions that are often found for IDPs. The picture that emerges from the studies discussed here is that conformational selection may play a role in the initial step of an IDP binding to its partner to a degree that corresponds to the amount of prestructuration present in its free-state ensemble. After the initial encounter, further inducedfit type rearrangements within the complex seem to occur nearly universally, from diffusion of a fully formed bound-state IDP conformation on the surface of its partner, as in the SeV N $\mathrm{NAIL}^{-}$ PX interaction (Schneider et al., 2015), to further folding of more complex structures, as apparent in, for example, the pKID-KIX or ACTR-NCBD interactions (Sugase et al., 2007a; Dogan et al., 2012).

Notably, the more complex a conformation adopted by an IDP in a complex, the more induced fit is likely to play a dominant role. While a single helix, given appropriate sequence composition such as $\mathrm{N}$-capping residues (Richardson and Richardson, 1988; Doig et al., 1997; Jensen et al., 2008), can form easily and quickly, more complex topologies such as the three-helix fold of ACTR in complex with NCBD are unlikely to contribute significantly to initial binding in a folded form. Especially in the absence of a stable hydrophobic core, their spontaneous formation appears exceedingly unlikely; in addition, the steric hindrance of binding of such a pre-formed conformation to its partner would be considerable. In case of pure conformational selection, dramatically reduced association rates would be expected (Zhou et al., 2012). Rather, IDPs seem to achieve fast association by their ability to form complex bound topologies and extended binding interfaces rapidly by virtue of their flexibility, once the binding partner presents a template for their induced folding beyond a pre-formed folded "nucleus." Coupled folding and binding of IDPs thus seems well described by the proposed "dock-and-coalesce" mechanism (Zhou et al., 2012) which, in terms classically used to describe protein folding, corresponds more to sequential structure formation as in diffusion-collision models (Karplus and Weaver, 1994), rather than nucleation-condensation mechanisms with concerted formation of secondary and tertiary structure (Itzhaki et al., 1995). Nevertheless, the variability observed in IDP binding mechanisms warrants caution in any such generalization.

In a broader sense, the observation that conformational selection and induced fit may be combined within a single pathway in a binding interaction suggests that the generic term "coupled folding and binding," often employed to leave open the question about whether binding precedes folding or vice versa, may actually be a quite accurate description of more complex interactions of IDPs with their partners. It has been stated that concurrent folding and binding would be extremely improbable since, already individually, folding and binding are low frequency stochastic events whose simultaneous occurrence would thus be even more rare (Hammes et al., 2009). However, this assumes that folding and binding are instantaneous, purely random events occurring on a static energy landscape, which is likely not the case. In particular, it has to be kept in mind that the energy landscape of interacting molecules changes while they approach each other. This has been underlined by a recent allatom MD study of the interaction of a folded protein, ubiquitin, with a short ubiquitin-interacting motif (UIM) peptide sequence (Long and Brüschweiler, 2011). Already at nanometer distance to the ligand, largely due to long-range electrostatic effects, it was found that the energy landscape of ubiquitin began to change, more and more favoring a preexisting energy well containing conformers similar to the bound state. It was pointed out that this interaction is well described by induced fit when considering the average structure of ubiquitin at any given protein-ligand distance; however, when considering the entire ensemble of ubiquitin conformations present at each point of the approach, conformational selection on a changing energy landscape is a more appropriate characterization. The overall mechanism was thus described as a "superposition" of conformational selection and induced fit (Long and Brüschweiler, 2011). Such a description may be well suited to explain the complex kinetics of folding and binding observed experimentally in IDPs, whose highly dynamic nature makes the need of ensemble descriptions even more evident (Jensen et al., 2014). Indeed, the term "conformational funneling" that we introduced in the context of the $\mathrm{SeV} \mathrm{N}_{\mathrm{TAIL}}$-PX interaction is conceptually very similar (Schneider et al., 2015; Gianni et al., 2016). This approach would also allow to transcend the likely too simplistic view of mutually exclusive, strictly sequential or strictly parallel conformational selection or induced fit pathways.

\section{Beyond Structure: Fuzzy Complexes}

While so far we have mostly discussed IDP binding interactions that involve individual binding sites and lead to well-defined complexes, the repertoire of binding mechanisms available to IDPs has been found to be much larger than that. So-called "fuzzy complexes" can retain considerable dynamics in the bound state (Tompa and Fuxreiter, 2008). In fact, the complex between $\mathrm{N}_{\mathrm{TAIL}}$ and PX in Sendai virus discussed above already provides one example, with the nonspecific initial complex of $\mathrm{N}_{\text {TAIL }}$ diffusing on the surface of PX actually being more populated than the final bound state (Schneider et al., 2015). This dynamic behavior has also been used as an explanation for the persistent line broadening observed in NMR spectra of this complex, even in presence of excess ligand. The disordered cyclin-dependent kinase inhibitor Sic1 has been found to interact with a single binding site on its receptor Cdc4 via multiple phosphorylated suboptimal binding sites that engage the partner in rapid exchange and only become transiently ordered upon interaction (Mittag et al., 2008). The requirement for each site to be phosphorylated for interaction, as well as the rapid equilibrium of several Sic1 sites exchanging on a single receptor binding site, leads to global high-affinity binding and a finely tunable, sensitive response of this interaction to Sic1 phosphorylation. An even more extreme example of dynamic binding is found in the interaction of phenylalanine-glycine(FG-) rich nucleoporins (FG-Nups) with nuclear transport receptors (NTRs) during their transit through the nuclear pore complex. A recent detailed investigation of the interaction of the NTR importin $\beta$ with a PxFG-rich domain of the FGNup Nup153 has demonstrated extremely rapid, concurrent 
binding of minimalistic Nup153 motifs (in principle, individual phenylalanine side chains) to importin $\beta$, while the overall disordered nature of Nup153 remained unperturbed, to the extent that backbone ${ }^{13} \mathrm{C}$ chemical shifts remain oblivious to the interactions mediated by their aromatic sidechain moieties (Milles et al., 2015). The unique properties of this multivalent interaction have been proposed to be at the core of rapid nuclear transport. Dynamic complexes of IDPs and multisite interactions thus seem to provide important advantages for rapid, yet sensitive and selective molecular recognition, with the avoidance of a large entropy loss upon binding likely being one of them. The hypothesis that an IDP interaction could be dynamic to such an extent that NMR spectra remain completely unaffected, as put forward for the dimerization of the T-cell receptor zeta subunit (Sigalov et al., 2007), has however not been confirmed by further experiments (Nourse and Mittag, 2014), suggesting that even "fuzzy complexes" are characterized by a transient local gain in structure within binding sites.

\section{CONCLUDING REMARKS}

IDPs often seem to escape concepts that attempt to unify and generalize their behavior. While some phenomena, such as fast association rates or moderate affinities, are found recurrently in IDP interactions, they are not generally associated with disorder, and IDPs appear to employ various combinations of conformational selection and induced fit mechanisms of binding, making use of both pre-formed structured elements and

\section{REFERENCES}

Arai, M., Ferreon, J. C., and Wright, P. E. (2012). Quantitative analysis of multisite protein-ligand interactions by NMR: binding of intrinsically disordered p53 transactivation subdomains with the TAZ2 domain of CBP. J. Am. Chem. Soc. 134, 3792-3803. doi: 10.1021/ja209936u

Arai, M., Sugase, K., Dyson, H. J., and Wright, P. E. (2015). Conformational propensities of intrinsically disordered proteins influence the mechanism of binding and folding. Proc. Natl. Acad. Sci. U.S.A. 112, 9614-9619. doi: 10.1073/pnas.1512799112

Barducci, A., Bussi, G., and Parrinello, M. (2008). Well-tempered metadynamics: a smoothly converging and tunable free-energy method. Phys. Rev. Lett. 100:20603. doi: 10.1103/PhysRevLett.100.020603

Bernasconi, C. F. (1976). Relaxation Kinetics. New York, NY: Academic Press.

Best, R. B., and Hummer, G. (2009). Optimized molecular dynamics force fields applied to the helix-coil transition of polypeptides. J. Phys. Chem. B 113, 9004-9015. doi: 10.1021/jp901540t

Bienkiewicz, E. A., Adkins, J. N., and Lumb, K. J. (2002). Functional consequences of preorganized helical structure in the intrinsically disordered cell-cycle inhibitor p27(Kip1). Biochemistry 41, 752-759. doi: 10.1021/bi015763t

Bodart, J.-F., Wieruszeski, J.-M., Amniai, L., Leroy, A., Landrieu, I., RousseauLescuyer, A., et al. (2008). NMR observation of Tau in Xenopus oocytes. J. Magn. Reson. 192, 252-257. doi: 10.1016/j.jmr.2008.03.006

Boehr, D. D., Nussinov, R., and Wright, P. E. (2009). The role of dynamic conformational ensembles in biomolecular recognition. Nat. Chem. Biol. 5, 789-796. doi: 10.1038/nchembio.232

Borcherds, W., Theillet, F.-X., Katzer, A., Finzel, A., Mishall, K. M., Powell, A. T., et al. (2014). Disorder and residual helicity alter p53-Mdm2 binding affinity and signaling in cells. Nat. Chem. Biol. 10, 1000-1002. doi: 10.1038/nchemb io. 1668 structural adaptation after binding. However, it should not be expected that IDPs are a homogeneous class of proteins; their mechanistic repertoire is large, as is the range of functions they are involved in. In that sense, IDPs should probably be regarded as less fundamentally different from folded proteins than they appear at first glance; after all, they are governed by the same fundamental laws of kinetics and thermodynamics. In recent years, techniques such as transient kinetics, NMR spectroscopy, and molecular simulations have considerably increased our knowledge about the mechanisms employed by IDPs to fulfill their functions. In particular, it has become increasingly evident that combining the results gained using these different techniques allows for additional mechanistic insight not easily obtained from the individual approaches alone. Further research in this exciting field should allow us to gain a more representative picture on what may or may not distinguish disorder from order in protein function.

\section{AUTHOR CONTRIBUTIONS}

All authors participated in writing of the manuscript and approved of its final version.

\section{ACKNOWLEDGMENTS}

We are grateful to Magnus Kjærgaard for providing Figures 2A,B.

Csermely, P., Palotai, R., and Nussinov, R. (2010). Induced fit, conformational selection and independent dynamic segments: an extended view of binding events. Trends Biochem. Sci. 35, 539-546. doi: 10.1016/j.tibs.2010.04.009

Daniels, K. G., Tonthat, N. K., McClure, D. R., Chang, Y.-C., Liu, X., Schumacher, M. A., et al. (2014). Ligand concentration regulates the pathways of coupled protein folding and binding. J. Am. Chem. Soc. 136, 822-825. doi: $10.1021 /$ ja4086726

Demarest, S. J., Martinez-Yamout, M., Chung, J., Chen, H., Xu, W., Dyson, H. J., et al. (2002). Mutual synergistic folding in recruitment of CBP/p300 by p160 nuclear receptor coactivators. Nature 415, 549-553. doi: 10.1038/415549a

De Sancho, D., and Best, R. B. (2012). Modulation of an IDP binding mechanism and rates by helix propensity and non-native interactions: association of HIF1 $\alpha$ with CBP. Mol. Biosyst. 8, 256-267. doi: 10.1039/C1MB05252G

De Vivo, M., Masetti, M., Bottegoni, G., and Cavalli, A. (2016). Role of molecular dynamics and related methods in drug discovery. J. Med. Chem. 59, 4035-4061. doi: 10.1021/acs.jmedchem.5b01684

Do, T. N., Choy, W.-Y., and Karttunen, M. (2014). Accelerating the conformational sampling of intrinsically disordered proteins. J. Chem. Theory Comput. 10, 5081-5094. doi: 10.1021/ct5004803

Dogan, J., Gianni, S., and Jemth, P. (2014). The binding mechanisms of intrinsically disordered proteins. Phys. Chem. Chem. Phys. 16, 6323-6331. doi: 10.1039/C3CP54226B

Dogan, J., and Jemth, P. (2014). Only kinetics can prove conformational selection. Biophys. J. 107, 1997-1998. doi: 10.1016/j.bpj.2014.08.037

Dogan, J., Jonasson, J., Andersson, E., and Jemth, P. (2015). Binding rate constants reveal distinct features of disordered protein domains. Biochemistry 54, 4741-4750. doi: 10.1021/acs.biochem.5b00520

Dogan, J., Mu, X., Engström, Å., and Jemth, P. (2013). The transition state structure for coupled binding and folding of disordered protein domains. Sci. Rep. 3:2076. doi: $10.1038 /$ srep02076 
Dogan, J., Schmidt, T., Mu, X., Engström, A., and Jemth, P. (2012). Fast association and slow transitions in the interaction between two intrinsically disordered protein domains. J. Biol. Chem. 287, 34316-34324. doi: 10.1074/jbc.M112.399436

Doig, A. J., MacArthur, M. W., Stapley, B. J., and Thornton, J. M. (1997). Structures of N-termini of helices in proteins. Protein Sci. 6, 147-155. doi: $10.1002 /$ pro.5560060117

Domene, C., Barbini, P., and Furini, S. (2015). Bias-exchange metadynamics simulations: an efficient strategy for the analysis of conduction and selectivity in ion channels. J. Chem. Theory Comput. 11, 1896-1906. doi: 10.1021/ct501053x

Dosnon, M., Bonetti, D., Morrone, A., Erales, J., di Silvio, E., Longhi, S., et al. (2015). Demonstration of a folding after binding mechanism in the recognition between the measles virus NTAIL and X domains. ACS Chem. Biol. 10, 795-802. doi: $10.1021 / \mathrm{cb} 5008579$

Dujardin, M., Madan, V., Montserret, R., Ahuja, P., Huvent, I., Launay, H., et al. (2015). A proline-tryptophan turn in the intrinsically disordered domain 2 of NS5A protein is essential for Hepatitis C virus RNA replication. J. Biol. Chem. 290, 19104-19120. doi: 10.1074/jbc.M115.644419

Dunker, A. K., Brown, C. J., Lawson, J. D., Iakoucheva, L. M., and Obradoviæ, Z. (2002). Intrinsic disorder and protein function. Biochemistry 41, 6573-6582. doi: 10.1021/bi012159+

Dunker, A. K., Lawson, J. D., Brown, C. J., Williams, R. M., Romero, P., Oh, J. S., et al. (2001). Intrinsically disordered protein. J. Mol. Graph. Model. 19, 26-59. doi: 10.1016/S1093-3263(00)00138-8

Dunker, A. K., Obradovic, Z., Romero, P., Garner, E. C., and Brown, C. J. (2000). Intrinsic protein disorder in complete genomes. Genome Inform. Ser. Workshop Genome Inform. 11, 161-171. Available online at: http://www.dabi.temple. edu/ zoran/papers/dunker00.pdf

Dyson, H. J., and Wright, P. E. (2002). Coupling of folding and binding for unstructured proteins. Curr. Opin. Struct. Biol. 12, 54-60. doi: 10.1016/S0959440X(02)00289-0

Dyson, H. J., and Wright, P. E. (2005). Intrinsically unstructured proteins and their functions. Nat. Rev. Mol. Cell Biol. 6, 197-208. doi: 10.1038/nrm1589

Eaton, W. A., Muñoz, V., Thompson, P. A., Chan, C. K., and Hofrichter, J. (1997). Submillisecond kinetics of protein folding. Curr. Opin. Struct. Biol. 7, 10-14.

Eigen, M., and Hammes, G. G. (1963). "Elementary steps in enzyme reactions (as studied by relaxation spectrometry)," in Advances in Enzymology - and Related Areas of Molecular Biology, ed F. F. Nord (Hoboken, NJ), 1-38. Available online at: http://doi.wiley.com/10.1002/9780470122709.ch1 (Accessed June 23, 2016).

Espinoza-Fonseca, L. M. (2009). Reconciling binding mechanisms of intrinsically disordered proteins. Biochem. Biophys. Res. Commun. 382, 479-482. doi: 10.1016/j.bbrc.2009.02.151

Fersht, A. R., and Sato, S. (2004). Phi-value analysis and the nature of proteinfolding transition states. Proc. Natl. Acad. Sci. U.S.A. 101, 7976-7981. doi: 10.1073/pnas.0402684101

Fuxreiter, M., Simon, I., Friedrich, P., and Tompa, P. (2004). Preformed structural elements feature in partner recognition by intrinsically unstructured proteins. J. Mol. Biol. 338, 1015-1026. doi: 10.1016/j.jmb.2004.03.017

Gambin, Y., VanDelinder, V., Ferreon, A. C. M., Lemke, E. A., Groisman, A., and Deniz, A. A. (2011). Visualizing a one-way protein encounter complex by ultrafast single-molecule mixing. Nat. Methods 8, 239-241. doi: $10.1038 /$ nmeth. 1568

Ganguly, D., Otieno, S., Waddell, B., Iconaru, L., Kriwacki, R. W., and Chen, J. (2012). Electrostatically accelerated coupled binding and folding of intrinsically disordered proteins. J. Mol. Biol. 422, 674-684. doi: 10.1016/j.jmb.2012. 06.019

Ganguly, D., Zhang, W., and Chen, J. (2013). Electrostatically accelerated encounter and folding for facile recognition of intrinsically disordered proteins. PLoS Comput. Biol. 9:e1003363. doi: 10.1371/journal.pcbi.1003363

Gianni, S., Dogan, J., and Jemth, P. (2014). Distinguishing induced fit from conformational selection. Biophys. Chem. 189, 33-39. doi: 10.1016/j.bpc.2014.03.003

Gianni, S., Dogan, J., and Jemth, P. (2016). Coupled binding and folding of intrinsically disordered proteins: what can we learn from kinetics? Curr. Opin. Struct. Biol. 36, 18-24. doi: 10.1016/j.sbi.2015.11.012

Gianni, S., Morrone, A., Giri, R., and Brunori, M. (2012). A folding-after-binding mechanism describes the recognition between the transactivation domain of cMyb and the KIX domain of the CREB-binding protein. Biochem. Biophys. Res. Commun. 428, 205-209. doi: 10.1016/j.bbrc.2012.09.112
Giri, R., Morrone, A., Toto, A., Brunori, M., and Gianni, S. (2013). Structure of the transition state for the binding of c-Myb and KIX highlights an unexpected order for a disordered system. Proc. Natl. Acad. Sci. U.S.A. 110, 14942-14947. doi: $10.1073 /$ pnas. 1307337110

Hammes, G. G., Chang, Y.-C., and Oas, T. G. (2009). Conformational selection or induced fit: a flux description of reaction mechanism. Proc. Natl. Acad. Sci. U.S.A. 106, 13737-13741. doi: 10.1073/pnas.0907195106

Han, M., Xu, J., Ren, Y., and Li, J. (2016). Simulation of coupled folding and binding of an intrinsically disordered protein in explicit solvent with metadynamics. J. Mol. Graph. Model. 68, 114-127. doi: 10.1016/j.jmgm.2016.06.015

Henriques, J., Cragnell, C., and Skepö, M. (2015). Molecular dynamics simulations of intrinsically disordered proteins: force field evaluation and comparison with experiment. J. Chem. Theory Comput. 11, 3420-3431. doi: 10.1021/ct501178z

Horn, H. W., Swope, W. C., Pitera, J. W., Madura, J. D., Dick, T. J., Hura, G. L., et al. (2004). Development of an improved four-site water model for biomolecular simulations: TIP4P-Ew. J. Chem. Phys. 120, 9665-9678. doi: 10.1063/1.1683075

Huang, Y., and Liu, Z. (2009). Kinetic advantage of intrinsically disordered proteins in coupled folding-binding process: a critical assessment of the "fly-casting" mechanism. J. Mol. Biol. 393, 1143-1159. doi: 10.1016/j.jmb.2009.09.010

Iakoucheva, L. M., Radivojac, P., Brown, C. J., O’Connor, T. R., Sikes, J. G., Obradovic, Z., et al. (2004). The importance of intrinsic disorder for protein phosphorylation. Nucleic Acids Res. 32, 1037-1049. doi: 10.1093/nar/gkh253

Iešmantavičius, V., Dogan, J., Jemth, P., Teilum, K., and Kjaergaard, M. (2014). Helical propensity in an intrinsically disordered protein accelerates ligand binding. Angew. Chem. Int. Ed. Engl. 53, 1548-1551. doi: 10.1002/anie.201307712

Iešmantavičius, V., Jensen, M. R., Ozenne, V., Blackledge, M., Poulsen, F. M., and Kjaergaard, M. (2013). Modulation of the intrinsic helix propensity of an intrinsically disordered protein reveals long-range helix-helix interactions. J. Am. Chem. Soc. 135, 10155-10163. doi: 10.1021/ja4045532

Irbäck, A., and Mohanty, S. (2006). PROFASI: a monte carlo simulation package for protein folding and aggregation. J. Comput. Chem. 27, 1548-1555. doi: $10.1002 /$ jcc. 20452

Ithuralde, R. E., Roitberg, A. E., and Turjanski, A. G. (2016). Structured and unstructured binding of an intrinsically disordered protein as revealed by atomistic simulations. J. Am. Chem. Soc. 138, 8742-8751. doi: 10.1021/jacs.6b02016

Itzhaki, L. S., Otzen, D. E., and Fersht, A. R. (1995). The structure of the transition state for folding of chymotrypsin inhibitor 2 analysed by protein engineering methods: evidence for a nucleation-condensation mechanism for protein folding. J. Mol. Biol. 254, 260-288. doi: 10.1006/jmbi.1995.0616

Japrung, D., Dogan, J., Freedman, K. J., Nadzeyka, A., Bauerdick, S., Albrecht, T., et al. (2013). Single-molecule studies of intrinsically disordered proteins using solid-state nanopores. Anal. Chem. 85, 2449-2456. doi: 10.1021/ac3035025

Jensen, M. R., and Blackledge, M. (2014). Testing the validity of ensemble descriptions of intrinsically disordered proteins. Proc. Natl. Acad. Sci. U.S.A. 111, E1557-E1558. doi: 10.1073/pnas.1323876111

Jensen, M. R., Communie, G., Ribeiro, E. A., Martinez, N., Desfosses, A., Salmon, L., et al. (2011). Intrinsic disorder in measles virus nucleocapsids. Proc. Natl. Acad. Sci. U.S.A. 108, 9839-9844. doi: 10.1073/pnas.1103270108

Jensen, M. R., Houben, K., Lescop, E., Blanchard, L., Ruigrok, R. W. H., and Blackledge, M. (2008). Quantitative conformational analysis of partially folded proteins from residual dipolar couplings: application to the molecular recognition element of Sendai virus nucleoprotein. J. Am. Chem. Soc. 130, 8055-8061. doi: 10.1021/ja801332d

Jensen, M. R., Zweckstetter, M., Huang, J., and Blackledge, M. (2014). Exploring free-energy landscapes of intrinsically disordered proteins at atomic resolution using NMR spectroscopy. Chem. Rev. 114, 6632-6660. doi: 10.1021/cr400688u

Johnson, K. A. (2008). Role of induced fit in enzyme specificity: a molecular forward/reverse switch. J. Biol. Chem. 283, 26297-26301. doi: 10.1074/jbc.R800034200

Karanicolas, J., and Brooks, C. L. (2002). The origins of asymmetry in the folding transition states of protein L and protein G. Protein Sci. 11, 2351-2361. doi: $10.1110 /$ ps.0205402

Karplus, M., and Weaver, D. L. (1994). Protein folding dynamics: the diffusioncollision model and experimental data. Protein Sci. 3, 650-668. doi: $10.1002 /$ pro. 5560030413 
Karush, F. (1950). Heterogeneity of the binding sites of bovine serum albumin. J. Am. Chem. Soc. 72, 2705-2713. doi: 10.1021/ja01162a099

Kiefhaber, T., Bachmann, A., and Jensen, K. S. (2012). Dynamics and mechanisms of coupled protein folding and binding reactions. Curr. Opin. Struct. Biol. 22, 21-29. doi: 10.1016/j.sbi.2011.09.010

Kim, Y. B., Kalinowski, S. S., and Marcinkeviciene, J. (2007). A pre-steady state analysis of ligand binding to human glucokinase: evidence for a preexisting equilibrium. Biochemistry 46, 1423-1431. doi: 10.1021/bi0617308

Kirschner, K., Eigen, M., Bittman, R., and Voigt, B. (1966). The binding of nicotinamide-adenine dinucleotide to yeast d-glyceraldehyde-3-phosphate dehydrogenase: temperature-jump relaxation studies on the mechanism of an allosteric enzyme. Proc. Natl. Acad. Sci. U.S.A. 56, 1661-1667. doi: 10.1073/pnas.56.6.1661

Kjaergaard, M., Teilum, K., and Poulsen, F. M. (2010). Conformational selection in the molten globule state of the nuclear coactivator binding domain of CBP. Proc. Natl. Acad. Sci. U.S.A. 107, 12535-12540. doi: 10.1073/pnas.1001693107

Koshland, D. E. (1958). Application of a theory of enzyme specificity to protein synthesis. Proc. Natl. Acad. Sci. U.S.A. 44, 98-104. doi: 10.1073/pnas.44.2.98

Kragelj, J., Palencia, A., Nanao, M. H., Maurin, D., Bouvignies, G., Blackledge, M., et al. (2015). Structure and dynamics of the MKK7-JNK signaling complex. Proc. Natl. Acad. Sci. U.S.A. 112, 3409-3414. doi: 10.1073/pnas.1419528112

Krieger, J. M., Fusco, G., Lewitzky, M., Simister, P. C., Marchant, J., Camilloni, C., et al. (2014). Conformational recognition of an intrinsically disordered protein. Biophys. J. 106, 1771-1779. doi: 10.1016/j.bpj.2014.03.004

Kussie, P. H., Gorina, S., Marechal, V., Elenbaas, B., Moreau, J., Levine, A. J., et al. (1996). Structure of the MDM2 oncoprotein bound to the p53 tumor suppressor transactivation domain. Science 274, 948-953. doi: 10.1126/science.274.5289.948

Lacy, E. R., Filippov, I., Lewis, W. S., Otieno, S., Xiao, L., Weiss, S., et al. (2004). p27 binds cyclin-CDK complexes through a sequential mechanism involving binding-induced protein folding. Nat. Struct. Mol. Biol. 11, 358-364. doi: $10.1038 /$ nsmb746

Laio, A., and Parrinello, M. (2002). Escaping free-energy minima. Proc. Natl. Acad. Sci. U.S.A. 99, 12562-12566. doi: 10.1073/pnas. 202427399

Lange, O. F., Lakomek, N.-A., Farès, C., Schröder, G. F., Walter, K. F. A., Becker, S., et al. (2008). Recognition dynamics up to microseconds revealed from an RDC-derived ubiquitin ensemble in solution. Science 320, 1471-1475. doi: $10.1126 /$ science. 1157092

Leyrat, C., Schneider, R., Ribeiro, E. A. Jr., Yabukarski, F., Yao, M., Gérard, F. C. A., et al. (2012). Ensemble structure of the modular and flexible full-length vesicular stomatitis virus phosphoprotein. J. Mol. Biol. 423, 182-197. doi: 10.1016/j.jmb.2012.07.003

Leyrat, C., Yabukarski, F., Tarbouriech, N., Ribeiro, E. A., Jensen, M. R., Blackledge, M., et al. (2011). Structure of the vesicular stomatitis virus $\mathrm{N}^{0}-\mathrm{P}$ complex. PLoS Pathog. 7:e1002248. doi: 10.1371/journal.ppat.1002248

Li, C., Pazgier, M., Li, C., Yuan, W., Liu, M., Wei, G., et al. (2010). Systematic mutational analysis of peptide inhibition of the p53-MDM2/MDMX interactions. J. Mol. Biol. 398, 200-213. doi: 10.1016/j.jmb.2010.03.005

Li, D.-W., and Brüschweiler, R. (2010). NMR-based protein potentials. Angew. Chem. Int. Ed. Engl. 49, 6778-6780. doi: 10.1002/anie.201001898

Lindorff-Larsen, K., Piana, S., Palmo, K., Maragakis, P., Klepeis, J. L., Dror, R. O., et al. (2010). Improved side-chain torsion potentials for the Amber ff99SB protein force field. Proteins 78, 1950-1958. doi: 10.1002/prot.22711

Lindorff-Larsen, K., Trbovic, N., Maragakis, P., Piana, S., and Shaw, D. E. (2012). Structure and dynamics of an unfolded protein examined by molecular dynamics simulation. J. Am. Chem. Soc. 134, 3787-3791. doi: $10.1021 / \mathrm{ja} 209931 \mathrm{w}$

Long, D., and Brüschweiler, R. (2011). In silico elucidation of the recognition dynamics of ubiquitin. PLoS Comput. Biol. 7:e1002035. doi: 10.1371/journal.pcbi.1002035

Ma, B., Kumar, S., Tsai, C. J., and Nussinov, R. (1999). Folding funnels and binding mechanisms. Protein Eng. 12, 713-720. doi: 10.1093/protein/12.9.713

Malatesta, F. (2005). The study of bimolecular reactions under non-pseudo-first order conditions. Biophys. Chem. 116, 251-256. doi: 10.1016/j.bpc.2005.04.006

Mercadante, D., Milles, S., Fuertes, G., Svergun, D. I., Lemke, E. A., and Gräter, F. (2015). Kirkwood-buff approach rescues overcollapse of a disordered protein in canonical protein force fields. J. Phys. Chem. B 119, 7975-7984. doi: 10.1021/acs.jpcb.5b03440
Mészáros, B., Tompa, P., Simon, I., and Dosztányi, Z. (2007). Molecular principles of the interactions of disordered proteins. J. Mol. Biol. 372, 549-561. doi: 10.1016/j.jmb.2007.07.004

Milles, S., Mercadante, D., Aramburu, I. V., Jensen, M. R., Banterle, N., Koehler, C., et al. (2015). Plasticity of an ultrafast interaction between nucleoporins and nuclear transport receptors. Cell 163, 734-745. doi: 10.1016/j.cell.2015.09.047

Mittag, T., Orlicky, S., Choy, W.-Y., Tang, X., Lin, H., Sicheri, F., et al. (2008). Dynamic equilibrium engagement of a polyvalent ligand with a single-site receptor. Proc. Natl. Acad. Sci. U.S.A. 105, 17772-17777. doi: 10.1073/pnas.0809222105

Mittermaier, A. K., and Kay, L. E. (2009). Observing biological dynamics at atomic resolution using NMR. Trends Biochem. Sci. 34, 601-611. doi: 10.1016/j.tibs.2009.07.004

Nourse, A., and Mittag, T. (2014). The cytoplasmic domain of the T-cell receptor zeta subunit does not form disordered dimers. J. Mol. Biol. 426, 62-70. doi: 10.1016/j.jmb.2013.09.036

Okamoto, Y. (2004). Generalized-ensemble algorithms: enhanced sampling techniques for Monte Carlo and molecular dynamics simulations. J. Mol. Graph. Model. 22, 425-439. doi: 10.1016/j.jmgm.2003.12.009

Oldfield, C. J., Meng, J., Yang, J. Y., Yang, M. Q., Uversky, V. N., and Dunker, A. K. (2008). Flexible nets: disorder and induced fit in the associations of p53 and 14-3-3 with their partners. BMC Genomics 9(Suppl. 1):S1. doi: 10.1186/14712164-9-S1-S1

Ostermeir, K., and Zacharias, M. (2013). Advanced replica-exchange sampling to study the flexibility and plasticity of peptides and proteins. Biochim. Biophys. Acta 1834, 847-853. doi: 10.1016/j.bbapap.2012.12.016

Palmer, A. G., Kroenke, C. D., and Loria, J. P. (2001). Nuclear magnetic resonance methods for quantifying microsecond-to-millisecond motions in biological macromolecules. Methods Enzymol. 339, 204-238. doi: 10.1016/S00766879(01)39315-1

Palmer, A. G., and Massi, F. (2006). Characterization of the dynamics of biomacromolecules using rotating-frame spin relaxation NMR spectroscopy. Chem. Rev. 106, 1700-1719. doi: 10.1021/cr0404287

Parker, D., Jhala, U. S., Radhakrishnan, I., Yaffe, M. B., Reyes, C., Shulman, A. I., et al. (1998). Analysis of an activator:coactivator complex reveals an essential role for secondary structure in transcriptional activation. Mol. Cell 2, 353-359. doi: 10.1016/S1097-2765(00)80279-8

Parker, D., Rivera, M., Zor, T., Henrion-Caude, A., Radhakrishnan, I., Kumar, A., et al. (1999). Role of secondary structure in discrimination between constitutive and inducible activators. Mol. Cell. Biol. 19, 5601-5607. doi: 10.1128/MCB.19.8.5601

Petros, A. M., Nettesheim, D. G., Wang, Y., Olejniczak, E. T., Meadows, R. P., Mack, J., et al. (2000). Rationale for Bcl-xL/Bad peptide complex formation from structure, mutagenesis, and biophysical studies. Protein Sci. 9, 2528-2534. doi: $10.1017 /$ S096183680000331X

Piana, S., Donchev, A. G., Robustelli, P., and Shaw, D. E. (2015). Water dispersion interactions strongly influence simulated structural properties of disordered protein states. J. Phys. Chem. B 119, 5113-5123. doi: 10.1021/jp508971m

Prakash, M. K. (2011). Insights on the role of (dis)order from protein-protein interaction linear free-energy relationships. J. Am. Chem. Soc. 133, 9976-9979. doi: $10.1021 / \mathrm{ja} 201500 \mathrm{z}$

Rauscher, S., Gapsys, V., Gajda, M. J., Zweckstetter, M., de Groot, B. L., and Grubmüller, H. (2015). Structural ensembles of intrinsically disordered proteins depend strongly on force field: a comparison to experiment. J. Chem. Theory Comput. 11, 5513-5524. doi: 10.1021/acs.jctc.5b00736

Richardson, J. S., and Richardson, D. C. (1988). Amino acid preferences for specific locations at the ends of alpha helices. Science 240, 1648-1652. doi: $10.1126 /$ science. 3381086

Rogers, J. M., Oleinikovas, V., Shammas, S. L., Wong, C. T., De Sancho, D., Baker, C. M., et al. (2014a). Interplay between partner and ligand facilitates the folding and binding of an intrinsically disordered protein. Proc. Natl. Acad. Sci. U.S.A. 111, 15420-15425. doi: 10.1073/pnas.1409122111

Rogers, J. M., Steward, A., and Clarke, J. (2013). Folding and binding of an intrinsically disordered protein: fast, but not "diffusion-limited." J. Am. Chem. Soc. 135, 1415-1422. doi: 10.1021/ja309527h

Rogers, J. M., Wong, C. T., and Clarke, J. (2014b). Coupled folding and binding of the disordered protein PUMA does not require particular residual structure. J. Am. Chem. Soc. 136, 5197-5200. doi: 10.1021/ja4125065 
Salmon, L., Nodet, G., Ozenne, V., Yin, G., Jensen, M. R., Zweckstetter, M., et al. (2010). NMR characterization of long-range order in intrinsically disordered proteins. J. Am. Chem. Soc. 132, 8407-8418. doi: 10.1021/jal $01645 \mathrm{~g}$

Salvi, N., Abyzov, A., and Blackledge, M. (2016). Multi-timescale dynamics in intrinsically disordered proteins from NMR relaxation and molecular simulation. J. Phys. Chem. Lett. 7, 2483-2489. doi: 10.1021/acs.jpclett.6b 00885

Schneider, R., Maurin, D., Communie, G., Kragelj, J., Hansen, D. F., Ruigrok, R. W. H., et al. (2015). Visualizing the molecular recognition trajectory of an intrinsically disordered protein using multinuclear relaxation dispersion NMR. J. Am. Chem. Soc. 137, 1220-1229. doi: 10.1021/ja5 $11066 \mathrm{q}$

Schuck, P., and Zhao, H. (2010). The role of mass transport limitation and surface heterogeneity in the biophysical characterization of macromolecular binding processes by SPR biosensing. Methods Mol. Biol. 627, 15-54. doi: 10.1007/978-1-60761-670-2_2

Shammas, S. L., Travis, A. J., and Clarke, J. (2013). Remarkably fast coupled folding and binding of the intrinsically disordered transactivation domain of cMyb to CBP KIX. J. Phys. Chem. B 117, 13346-13356. doi: 10.1021/jp40 $4267 \mathrm{e}$

Shammas, S. L., Travis, A. J., and Clarke, J. (2014). Allostery within a transcription coactivator is predominantly mediated through dissociation rate constants. Proc. Natl. Acad. Sci. U.S.A. 111, 12055-12060. doi: 10.1073/pnas.14058 15111

Shoemaker, B. A., Portman, J. J., and Wolynes, P. G. (2000). Speeding molecular recognition by using the folding funnel: the fly-casting mechanism. Proc. Natl. Acad. Sci. U.S.A. 97, 8868-8873. doi: 10.1073/pnas.160259697

Sickmeier, M., Hamilton, J. A., LeGall, T., Vacic, V., Cortese, M. S., Tantos, A., et al. (2007). DisProt: the database of disordered proteins. Nucleic Acids Res. 35, D786-D793. doi: 10.1093/nar/gkl893

Sigalov, A. B., Zhuravleva, A. V., and Orekhov, V. Y. (2007). Binding of intrinsically disordered proteins is not necessarily accompanied by a structural transition to a folded form. Biochimie 89, 419-421. doi: 10.1016/j.biochi.2006.11.003

Smith, W. W., Ho, P.-Y., and O'Hern, C. S. (2014). Calibrated langevin-dynamics simulations of intrinsically disordered proteins. Phys. Rev. E Stat. Nonlin. Soft Matter. Phys. 90:42709. doi: 10.1103/PhysRevE.90.042709

Spolar, R. S., and Record, M. T. (1994). Coupling of local folding to site-specific binding of proteins to DNA. Science 263, 777-784. doi: $10.1126 /$ science. 8303294

Staneva, I., Huang, Y., Liu, Z., and Wallin, S. (2012). Binding of two intrinsically disordered peptides to a multi-specific protein: a combined Monte Carlo and molecular dynamics study. PLoS Comput. Biol. 8:e1002682. doi: 10.1371/journal.pcbi.1002682

Sugase, K., Dyson, H. J., and Wright, P. E. (2007a). Mechanism of coupled folding and binding of an intrinsically disordered protein. Nature 447, 1021-1025. doi: 10.1038 /nature 05858

Sugase, K., Lansing, J. C., Dyson, H. J., and Wright, P. E. (2007b). Tailoring relaxation dispersion experiments for fast-associating protein complexes. J. Am. Chem. Soc. 129, 13406-13407. doi: 10.1021/ja0762238

Tai, K. (2004). Conformational sampling for the impatient. Biophys. Chem. 107, 213-220. doi: 10.1016/j.bpc.2003.09.010

Takada, S., Kanada, R., Tan, C., Terakawa, T., Li, W., and Kenzaki, H. (2015). Modeling structural dynamics of biomolecular complexes by coarse-grained molecular simulations. Acc. Chem. Res. 48, 3026-3035. doi: 10.1021/acs.accounts.5b00338

Theillet, F.-X., Binolfi, A., Bekei, B., Martorana, A., Rose, H. M., Stuiver, M., et al. (2016). Structural disorder of monomeric $\alpha$-synuclein persists in mammalian cells. Nature 530, 45-50. doi: 10.1038/nature16531

Tompa, P. (2002). Intrinsically unstructured proteins. Trends Biochem. Sci. 27, 527-533. doi: 10.1016/S0968-0004(02)02169-2

Tompa, P., and Fuxreiter, M. (2008). Fuzzy complexes: polymorphism and structural disorder in protein-protein interactions. Trends Biochem. Sci. 33, 2-8. doi: 10.1016/j.tibs.2007.10.003

Tompa, P., Szász, C., and Buday, L. (2005). Structural disorder throws new light on moonlighting. Trends Biochem. Sci. 30, 484-489. doi: 10.1016/j.tibs.2005. 07.008
Tummino, P. J., and Copeland, R. A. (2008). Residence time of receptor-ligand complexes and its effect on biological function. Biochemistry 47, 5481-5492. doi: $10.1021 /$ bi8002023

Ueda, Y., Taketomi, H., and Gō, N. (1978). Studies on protein folding, unfolding, and fluctuations by computer simulation. II. A. Threedimensional lattice model of lysozyme. Biopolymers 17, 1531-1548. doi: 10.1002/bip.1978.360170612

Uversky, V. N. (2002). Natively unfolded proteins: a point where biology waits for physics. Protein Sci. 11, 739-756. doi: 10.1110/ps.4210102

Uversky, V. N., Oldfield, C. J., and Dunker, A. K. (2008). Intrinsically disordered proteins in human diseases: introducing the D2 concept. Annu. Rev. Biophys. 37, 215-246. doi: 10.1146/annurev.biophys.37.032807.1 25924

Vallurupalli, P., Bouvignies, G., and Kay, L. E. (2012). Studying "invisible" excited protein states in slow exchange with a major state conformation. J. Am. Chem. Soc. $134,8148-8161$. doi: 10.1021/ja3001419

Vogt, A. D., and Di Cera, E. (2012). Conformational selection or induced fit? A critical appraisal of the kinetic mechanism. Biochemistry 51, 5894-5902. doi: 10.1021/bi3006913

Vogt, A. D., and Di Cera, E. (2013). Conformational selection is a dominant mechanism of ligand binding. Biochemistry 52, 5723-5729. doi: $10.1021 /$ bi400929b

Wang, L.-P., Martinez, T. J., and Pande, V. S. (2014). Building force fields: an automatic, systematic, and reproducible approach. J. Phys. Chem. Lett. 5, 1885-1891. doi: 10.1021/jz500737m

Wang, W., Ye, W., Jiang, C., Luo, R., and Chen, H.-F. (2014). New force field on modeling intrinsically disordered proteins. Chem. Biol. Drug Des. 84, 253-269. doi: $10.1111 /$ cbdd. 12314

Wang, Y., Chu, X., Longhi, S., Roche, P., Han, W., Wang, E., et al. (2013). Multiscaled exploration of coupled folding and binding of an intrinsically disordered molecular recognition element in measles virus nucleoprotein. Proc. Natl. Acad. Sci. U.S.A. 110, E3743-E3752. doi: 10.1073/pnas.13083 81110

Wells, M., Tidow, H., Rutherford, T. J., Markwick, P., Jensen, M. R., Mylonas, E., et al. (2008). Structure of tumor suppressor p53 and its intrinsically disordered N-terminal transactivation domain. Proc. Natl. Acad. Sci. U.S.A. 105, 5762-5767. doi: 10.1073/pnas.0801353105

Wong, I., Patel, S. S., and Johnson, K. A. (1991). An induced-fit kinetic mechanism for DNA replication fidelity: direct measurement by single-turnover kinetics. Biochemistry 30, 526-537. doi: 10.1021/bi00216a030

Wright, P. E., and Dyson, H. J. (1999). Intrinsically unstructured proteins: reassessing the protein structure-function paradigm. J. Mol. Biol. 293, 321-331. doi: 10.1006/jmbi.1999.3110

Wright, P. E., and Dyson, H. J. (2015). Intrinsically disordered proteins in cellular signalling and regulation. Nat. Rev. Mol. Cell Biol. 16, 18-29. doi: $10.1038 / \mathrm{nrm} 3920$

Ye, W., Ji, D., Wang, W., Luo, R., and Chen, H.-F. (2015). Test and evaluation of ff99IDPs force field for intrinsically disordered proteins. J. Chem. Inf. Model. 55, 1021-1029. doi: 10.1021/acs.jcim.5b00043

Zerze, G. H., Miller, C. M., Granata, D., and Mittal, J. (2015). Free energy surface of an intrinsically disordered protein: comparison between temperature replica exchange molecular dynamics and bias-exchange metadynamics. J. Chem. Theory Comput. 11, 2776-2782. doi: 10.1021/acs.jctc.5b 00047

Zhou, H.-X. (2012). Intrinsic disorder: signaling via highly specific but shortlived association. Trends Biochem. Sci. 37, 43-48. doi: 10.1016/j.tibs.2011. 11.002

Zhou, H.-X., and Bates, P. A. (2013). Modeling protein association mechanisms and kinetics. Curr. Opin. Struct. Biol. 23, 887-893. doi: 10.1016/j.sbi.2013.06.014

Zhou, H.-X., Pang, X., and Lu, C. (2012). Rate constants and mechanisms of intrinsically disordered proteins binding to structured targets. Phys. Chem. Chem. Phys. 14, 10466-10476. doi: 10.1039/c2cp41196b

Zondlo, S. C., Lee, A. E., and Zondlo, N. J. (2006). Determinants of specificity of MDM2 for the activation domains of p53 and p65: proline27 disrupts the MDM2-binding motif of p53. Biochemistry 45, 11945-11957. doi: 10.1021/bi060309g 
Zor, T., De Guzman, R. N., Dyson, H. J., and Wright, P. E. (2004). Solution structure of the KIX domain of CBP bound to the transactivation domain of c-Myb. J. Mol. Biol. 337, 521-534. doi: 10.1016/j.jmb.2004. 01.038

Zor, T., Mayr, B. M., Dyson, H. J., Montminy, M. R., and Wright, P. E. (2002). Roles of phosphorylation and helix propensity in the binding of the KIX domain of CREB-binding protein by constitutive (c-Myb) and inducible (CREB) activators. J. Biol. Chem. 277, 42241-42248. doi: 10.1074/jbc.M2073 61200

Zuiderweg, E. R. P. (2002). Mapping protein-protein interactions in solution by NMR spectroscopy. Biochemistry 41, 1-7. doi: 10.1021/bi011870b
Conflict of Interest Statement: The authors declare that the research was conducted in the absence of any commercial or financial relationships that could be construed as a potential conflict of interest.

Copyright (C) 2016 Mollica, Bessa, Hanoulle, Jensen, Blackledge and Schneider. This is an open-access article distributed under the terms of the Creative Commons Attribution License (CC BY). The use, distribution or reproduction in other forums is permitted, provided the original author(s) or licensor are credited and that the original publication in this journal is cited, in accordance with accepted academic practice. No use, distribution or reproduction is permitted which does not comply with these terms. 